\title{
A Subpopulation of Amygdala Neurons Mediates the Affective Component of Itch
}

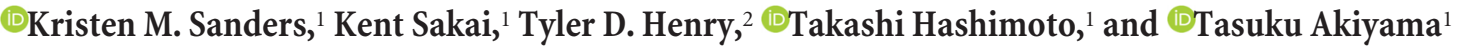 \\ ${ }^{1}$ Department of Dermatology \& Cutaneous Surgery and Miami Itch Center, University of Miami Miller School of Medicine, Miami, Florida 33136, \\ and ${ }^{2}$ Department of Biology, Temple University, Philadelphia, Pennsylvania 19122
}

Itch consists of both sensory and affective components. For chronic itch patients, the affective component of itch affects both quality of life (leading to psychological comorbidities) and disease prognosis (by promoting scratching of itchy skin). We found that acute itch stimuli, such as histamine, induced anxiety-like behavior and increased activity (c-Fos expression) in the amygdala in adult male C57BL/6 mice. Itch stimuli also increased activity in projection areas to the amygdala, suggesting that these regions form a circuit for affective itch processing. Electrophysiological characterization of histamine-responsive amygdala neurons showed that this population was active on a behaviorally relevant timescale and partially overlapped with pain signaling. Selective optogenetic activation of histamine-responsive amygdala neurons in adult male and female Fos:CreER ${ }^{\mathrm{T} 2} ; \mathrm{R}_{2} 6^{\mathrm{Ai} 14}$ mice using the Targeted Recombination in Active Populations system enhanced both scratching and anxiety-like behavior. These results highlight the importance of itch-responsive amygdala neurons in modulating itch-related affect and behavior.

Key words: itch; mouse; optogenetics; pruritus

\section{Significance Statement}

The sensation of itch includes an affective component that leads to stress and anxiety in chronic itch patients. We investigated the neuronal basis of affective itch in mice, with a focus on the amygdala, the key brain region for the generation of anxiety. A subpopulation of amygdala neurons responded to itch stimuli such as histamine. Optogenetic activation of histamine-responsive amygdala neurons affected both scratching and anxiety-like behavior. Therefore, this population appears to be important for mediating the affective component of itch.

\section{Introduction}

Itch, like pain, is composed of dissociable modalities, including sensory, discriminatory, motivational, and affective components. The affective component of itch can be experienced by anyone; by definition, itch is "unpleasant" and drives scratching behavior as a means of relief (Rothman, 1941). However, in individuals with chronic itch, the affective component contributes, over time, to mental distress and psychiatric comorbidities (Schneider et al., 2006; Ferm et al., 2010). In particular, chronic itch patients are vulnerable to a vicious cycle of itch and anxiety (Sanders and

Received Oct. 24, 2018; revised Feb. 19, 2019; accepted Feb. 21, 2019.

Author contributions: K.M.S., K.S., T.D.H., T.H., and T.A. performed research; K.M.S., K.S., and T.A. analyzed data; K.M.S. wrote the first draft of the paper; K.M.S., K.S., T.D.H., T.H., and T.A. edited the paper; K.M.S. and T.A. wrote the paper; T.A. designed research.

This work was supported by Grants from the National Institutes of Health (R00AR063228 and R01AR074062) to T.A. We thank Christian Albornoz (Temple University) for assistance with pilot immunohistochemistry studies and Kevin Johnson (University of Miami) for his generous technical support.

The authors declare no competing financial interests.

Correspondence should be addressed to Tasuku Akiyama at takiyama@miami.edu.

https://doi.org/10.1523/JNEUROSCI.2759-18.2019

Copyright $\odot 2019$ the authors
Akiyama, 2018). Itch evokes increased anxiety; in turn, stress and anxiety can exacerbate itch. Though chronic itch patients commonly use scratching to cope with itch and stress (Schut et al., 2015), this behavior provokes inflammation and damages the skin barrier, eventually worsening the underlying disease. Therefore, a mechanistic understanding of the itch-anxiety cycle is greatly needed to treat chronic itch and its affective symptoms.

Brain imaging studies have revealed limbic activations during itch that may be related to its affective component. The amygdala, the major center for generation of anxiety and fear, was activated by both histamine-dependent and -independent itch stimuli but deactivated by scratching (Papoiu et al., 2012, 2013; Vierow et al., 2015). Additionally, it has been reported that injection of a $\mathrm{GABA}_{\mathrm{A}}$ agonist into the central amygdala (CeA) suppressed acute and chronic itch in rats (Chen et al., 2016). Recent studies have identified populations of amygdala neurons that respond to other emotionally valent (appetitive or aversive) sensory stimuli, such as olfactory and taste cues (Xiu et al., 2014; Kim et al., 2016, 2017), and the amygdala is known to mediate the affective processing of pain (Neugebauer, 2015). However, to date there has 
been little investigation into the role of the amygdala in the affective processing of itch.

The amygdala receives nociceptive input from many brain regions and integrates this information to stimulate a behavioral response. Signals from the parabrachial nucleus (PBN) to the CeA are known to encode threat or danger signals and provoke fear-related behavior (Han et al., 2015; Campos et al., 2018). The PBN receives direct spinal input of itch signals (Mu et al., 2017) and therefore may convey itch information to the amygdala. The midcingulate cortex (MCC) plays a role in evaluating threat and coordinating an appropriate motor response and has reciprocal connections with the basolateral amygdala (BLA; Shackman et al., 2011). The MCC is also consistently active in fMRI studies of itch (Papoiu et al., 2012; Vierow et al., 2015). The medial prefrontal cortex (mPFC) has reciprocal connections with the BLA that promote either fear conditioning or fear extinction (Cho et al., 2013; Duvarci and Pare, 2014). As such, the mPFC may regulate itch-evoked activity in the amygdala.

We found that the amygdala, PBN, MCC, and mPFC were significantly activated by acute itch stimuli and therefore may serve as hubs of an "affective itch circuit" that drives anxiety-like behavior and contributes to the scratch response. Using in vivo single-unit electrophysiology, we confirmed the presence of itchresponsive neurons in the amygdala and characterized their firing properties in response to multiple pruritic and painful stimuli. Finally, using recently developed genetic and optogenetic techniques, we specifically activated itch-responsive neurons in the amygdala and observed that this activation enhanced itch- and anxiety-related behavior.

\section{Materials and Methods}

Mice. All procedures were approved by the Institutional Animal Care and Use Committees of Temple University and the University of Miami. For behavior testing, c-Fos immunohistochemistry, and electrophysiology experiments, male C57BL/6J mice (The Jackson Laboratory) were group housed (2-5 per cage), given standard food and water ad libitum, and maintained under a $12 \mathrm{~h}$ light cycle (06:00 lights on, 18:00 lights off). For sex comparison experiments, female mice were kept under the same housing conditions. All mice were acclimated for 1 week to the animal facility. Following this, all mice were habituated to handling by the experimenter, intradermal PBS and pruritogen injection, and to the testing room. A small area of fur on the rostral back was shaved for intradermal injections. At the time of testing, mice were 12-16 weeks old (weight $20-35 \mathrm{~g})$.

For retrograde virus experiments, female R26 ${ }^{\mathrm{Ail} 4}$ mice (The Jackson Laboratory) were maintained under the same housing conditions as the C57BL/6 mice. At the time of testing, mice were 9 months old (weight $39-41 \mathrm{~g})$. For optogenetics experiments, Fos:CreER ${ }^{\mathrm{T} 2}$ transgenic mice (The Jackson Laboratory) were mated with R26 ${ }^{\mathrm{Ai} 14}$ mice. Fos:CreER ${ }^{\mathrm{T} 2}$; R26 ${ }^{\text {Ail } 4}$ mice were group-housed by sex and maintained under the same housing conditions as the C57BL/6 mice. Adult male and female mice were used for experiments beginning at 10 weeks of age (weight 24-42 g).

Mice were randomly assigned to experimental groups. Mice were typically used for a battery of behavioral tests, with a 1 week break between each test. Mice that received capsaicin injections were not used for any further experiments, due to the potential for capsaicin to cause peripheral nerve damage (Simone et al., 1998). In every experiment, care was taken to minimize external sources of stress to the mice.

Scratching behavior. All behavioral testing was conducted between 9:00 A.M. and 6:00 P.M. Each apparatus was constructed of acrylic plastic (Interstate Plastics) and cleaned with $70 \%$ ethanol between mice to minimize odor effects. Each mouse was habituated to the recording chamber $(15 \times 15 \mathrm{~cm})$ before testing.

Histamine (50 $\mu \mathrm{g} / 10 \mu \mathrm{l}$; Sigma-Aldrich), chloroquine diphosphate salt (100 $\mu \mathrm{g} / 10 \mu \mathrm{l}$; Sigma-Aldrich), serotonin hydrochloride (10 $\mu \mathrm{g} / 10$ $\mu \mathrm{l}$; Alfa Aesar) or PBS vehicle was injected intradermally into the shaved rostral back skin of C57BL/6J mice. Injections were made using a $30 \mathrm{G}$ needle connected to a microsyringe (Hamilton) via PE-50 tubing (Instech Laboratories). Behavior was video recorded for $30 \mathrm{~min}$. The number of scratch bouts was analyzed in $5 \mathrm{~min}$ bins by a trained observer blinded to the treatment condition. One scratch bout was defined as one or more rapid back-and-forth hindpaw motions directed toward and contacting the injection site, ending with licking or biting of the toes or placement of the hindpaw on the floor. Hindpaw movements directed away from the injection site (e.g., ear-scratching) and grooming movements were not counted (Akiyama et al., 2009b).

Conditioned place aversion. Conditioned place aversion (CPA) was tested in plastic boxes $(12 \mathrm{~cm}$ wide $\times 29 \mathrm{~cm}$ long $\times 30.5 \mathrm{~cm}$ high) composed of two halves distinguished by wall pattern (vertical or horizontal stripes) and metal floor texture (bars or holes). A $10 \mathrm{~d}$ protocol was used, after King et al. (2009). On Days 1-3, each mouse was placed in a box for $30 \mathrm{~min}$ and could freely move between the two halves. For the next $6 \mathrm{~d}$, a divider was inserted into the box to confine the mouse to one half at a time. On Days 4, 6, and 8, the mouse received an intradermal injection of PBS vehicle into the rostral back and then was placed in one half of the box for $30 \mathrm{~min}$. PBS injection was used, as by Mu and Sun (2017), to control for any aversion induced by the intradermal injection process itself. On Days 5, 7, and 9, the mouse was injected with histamine $(50 \mu \mathrm{g} / 10 \mu \mathrm{l})$ or serotonin $(10 \mu \mathrm{g} / 10 \mu \mathrm{l})$ and placed in the other half of the box for $30 \mathrm{~min}$. The wall pattern and floor texture associated with the pruritogen/PBS were randomly assigned but consistent for each mouse across the experimental timeline. On Day 10, the divider was removed, and the mouse was placed back in the box for $30 \mathrm{~min}$ and allowed to move freely between the two sides.

Mice were video recorded while in the boxes, and videos were analyzed by a trained observer blinded to the treatment group. On Days 3 and 10 (at baseline and after conditioning), the time spent in each half of the box was determined. A mouse was considered to have entered one half of the box when all four paws were placed on the floor in that half. A decrease in time spent on the itch-conditioned side was considered a measure of aversion to itch.

Elevated plus maze. The elevated plus maze (EPM) was composed of four arms $(4.5 \mathrm{~cm}$ wide $\times 30 \mathrm{~cm}$ long): two opposing "closed arms" surrounded on three sides with transparent, $18 \mathrm{~cm}$ high walls; two opposing "open arms" with $1 \mathrm{~cm}$ high ledges to prevent the mice from falling; and a central square. The entire apparatus was raised $32 \mathrm{~cm}$ from the floor. To reduce baseline anxiety (Post et al., 2011), testing was conducted under red light $(\sim 20$ lux $)$ except in optogenetics experiments, which were conducted under normal lighting conditions ( $\sim 100$ lux).

Each mouse was weighed and acclimated to the testing room for at least $30 \mathrm{~min}$ before testing. To assess the effects of histaminergic and non-histaminergic pruritogens on anxiety-like behavior, histamine (50 $\mu \mathrm{g} / 10 \mu \mathrm{l})$, chloroquine $(100 \mu \mathrm{g} / 10 \mu \mathrm{l})$, and serotonin $(10 \mu \mathrm{g} / 10 \mu \mathrm{l})$ were tested versus PBS vehicle injection. For a comparative pain stimulus, capsaicin (30 $\mu \mathrm{g} / 10 \mu \mathrm{l}$, Enzo Life Sciences) was tested versus 7\% Tween 80 in PBS ("PBS-Tween") vehicle.

Each mouse received an intradermal injection into the rostral back skin and was returned to the home cage for $5 \mathrm{~min}$ to reduce anxiety associated with injection and to allow time for itch sensation to develop. Then, each mouse was placed into the center square of the EPM, facing an open arm, and video recorded for $10 \mathrm{~min}$. Videos were analyzed by a trained observer blinded to the treatment group. A mouse was considered to have entered an arm when all four paws were placed on the floor in that arm. Decreased time in open arms was a considered measure of anxiety-like behavior, and the total number of entries was used as a general measure of locomotion (Rodgers and Johnson, 1995). Time spent in closed arms and the center square were also reported to provide a more complete picture of mouse behavior during the test.

Open-field test. The open-field test (OFT) apparatus consisted of a large, square box $(60 \mathrm{~cm}$ long $\times 60 \mathrm{~cm}$ wide $\times 40 \mathrm{~cm}$ high $)$ with lines dividing the floor into 16 equal squares. The 12 squares adjacent to the wall were considered "peripheral", whereas the 4 squares in the middle of the floor were considered "central". Testing was conducted under red light, except in optogenetics experiments, which were conducted under normal lighting. 
A

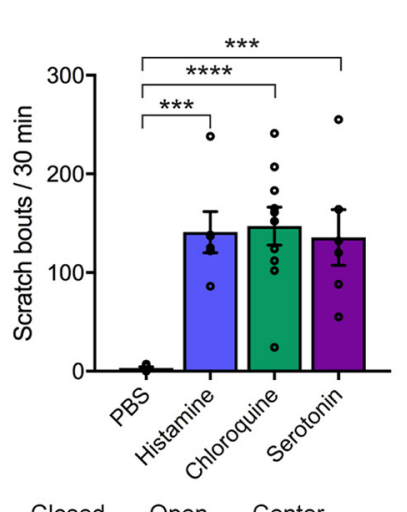

B

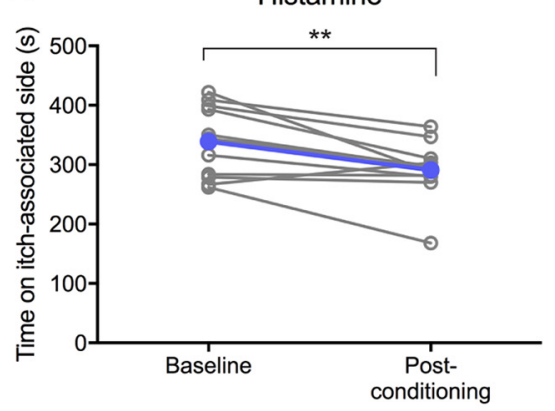

Serotonin

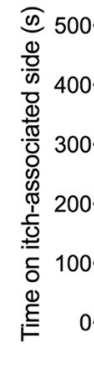

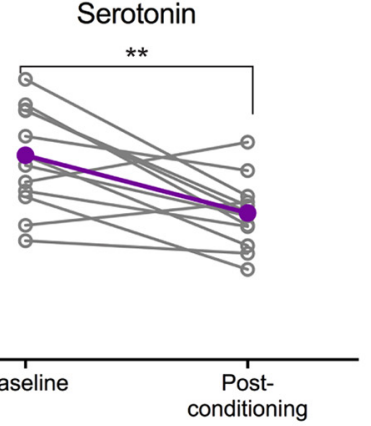
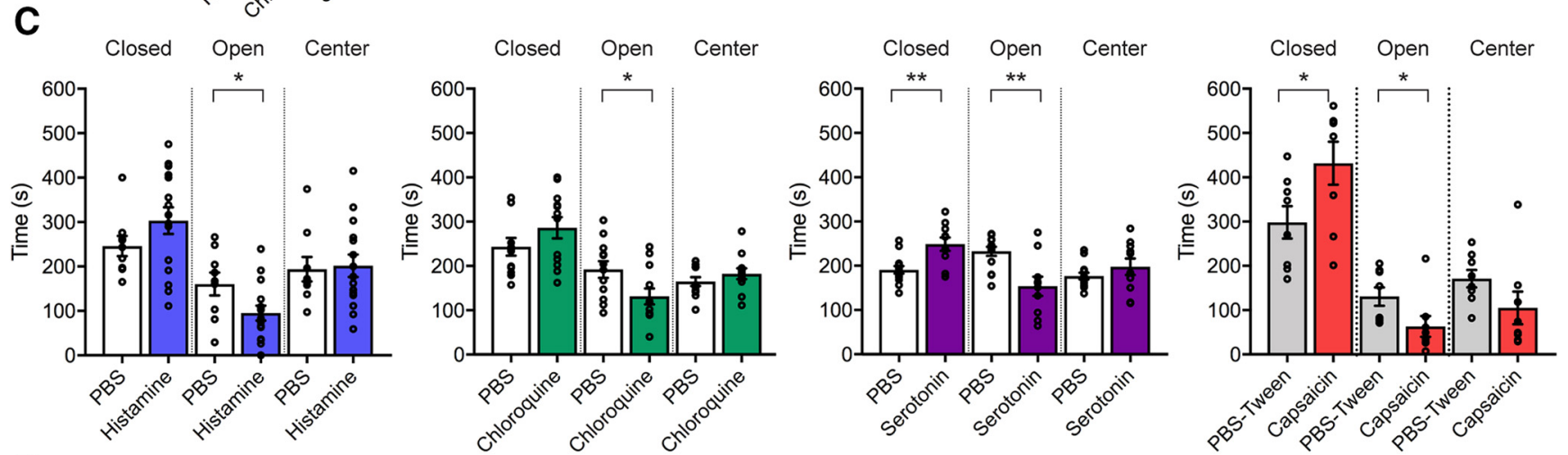

D
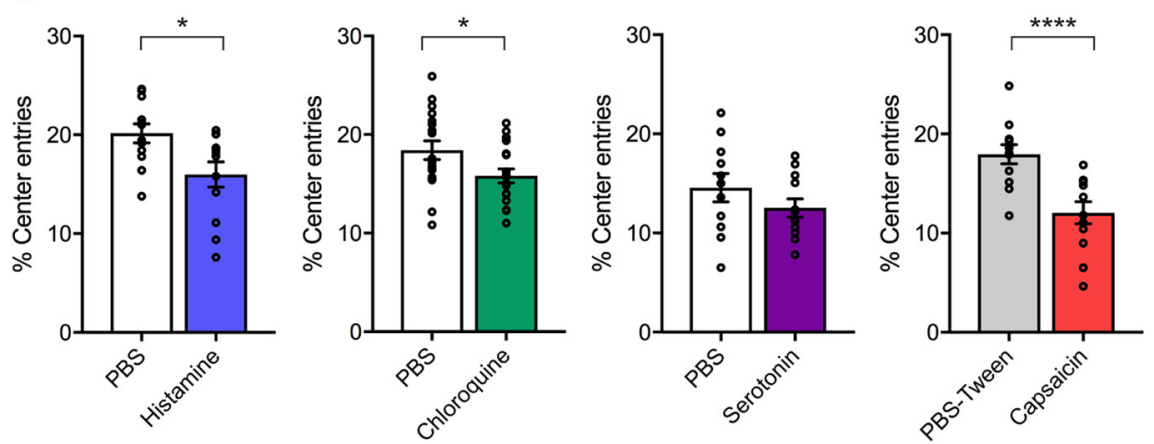

Figure 1. Acute itch stimuli evoke anxiety-like behavior in mice. $A$, Intradermal injections of histamine, chloroquine, and serotonin (but not PBS vehicle) provoked robust scratching behavior $\left(n=6-10 /\right.$ group). Histamine, $t_{(10)}=6.596, p<0.0001$; chloroquine, $t_{(14)}=5.727, p<0.0001$; serotonin, $t_{(10)}=4.677, p=0.0009$. $B$, Histamine and serotonin induced (PA, indicating that these stimuli are aversive to mice $\left(n=11-12 /\right.$ experiment). Gray lines indicate individual mice. Colored lines indicate averages. Histamine, $t_{(10)}=3.365, p=0.0072 ;$ serotonin, $t_{(11)}=3.616, p=$ 0.0041. C, On the EPM, histamine, chloroquine, and serotonin reduced open arm time compared with PBS, suggesting that acute itch causes thigmotaxis, an anxiety-like behavior. Capsaicin, a painful stimulus, induced a similar pattern of thigmotaxis compared with its vehicle, PBS-Tween $\left(n=8-15 /\right.$ group). Histamine, $t_{(22)}=2.209, p=0.0379 ;$ chloroquine, $t_{(22)}=2.325, p=0.0297$; serotonin, $t_{(22)}=3.645, p=0.0014$; capsaicin, $t_{(14)}=2.156, p=0.0490$. D , On the OFT, histamine, chloroquine, and capsaicin induced a reduction in the percentage of center square entries, another measure of thigmotaxis $\left(n=11-18 /\right.$ group). Histamine, $t_{(22)}=2.608, p=0.0160$; chloroquine, $t_{(34)}=2.198, p=0.0349$; serotonin, $t_{(21)}=1.225, p=0.2343$; capsaicin, $t_{(22)}=4.016$, $p=0.0006$. Data in $\boldsymbol{A}, \boldsymbol{C}$, and $\boldsymbol{D}$ are shown as mean \pm SEM. ${ }^{*} p<0.05,{ }^{* *} p<0.01,{ }^{* * *} p<0.001,{ }^{* * * *} p<0.0001$ for unpaired $t$ test versus vehicle $(\boldsymbol{A}, \boldsymbol{C}, \boldsymbol{D})$ or paired $t$ test $(\boldsymbol{B})$.

As in the EPM, each mouse was weighed and acclimated to the testing room for at least $30 \mathrm{~min}$ before testing. Each mouse received an intradermal injection of histamine $(50 \mu \mathrm{g} / 10 \mu \mathrm{l})$, chloroquine $(100 \mu \mathrm{g} / 10 \mu \mathrm{l})$, serotonin $(10 \mu \mathrm{g} / 10 \mu \mathrm{l})$, PBS, capsaicin $(30 \mu \mathrm{g} / 10 \mu \mathrm{l})$, or PBS-Tween into the rostral back skin and was returned to the home cage for $5 \mathrm{~min}$. Then, each mouse was placed into a corner of the OFT, facing parallel to a wall, and video recorded for $10 \mathrm{~min}$. Videos were analyzed by a trained observer blinded to the treatment group. The number of entries into central and peripheral squares were counted. A mouse was considered to have entered a square when all four paws were placed on the floor in that square. Decreased percentage entries into central squares (central/total) was considered a measure of anxiety-like behavior (Takahashi et al., 2006). The total number of square entries was used as a general measure of locomotion.

c-Fos immunohistochemistry. Two days before the experiment, C57BL/6J mice had the toenails on their hindpaws lightly trimmed to avoid the expression of c-Fos because of pain from scratching. On the experiment day, mice were weighed and habituated to the experiment room for $30 \mathrm{~min}$. Then, each mouse received an intradermal injection of PBS, histamine $(50 \mu \mathrm{g} / 10 \mu \mathrm{l})$, chloroquine $(100 \mu \mathrm{g} / 10 \mu \mathrm{l})$, serotonin $(10 \mu \mathrm{g} / 10 \mu \mathrm{l})$, PBS-Tween, or capsaicin $(30 \mu \mathrm{g} / 10 \mu \mathrm{l})$ into the rostral back skin. Following injection, each mouse was transferred to a quiet, familiar behavior chamber for $2 \mathrm{~h}$ to allow for peak injectioninduced c-Fos expression and to minimize neuronal activity from external stress (Akiyama et al., 2009b; Gao and Ji, 2009). After 2 h, mice were anesthetized with intraperitoneal (i.p.) sodium pentobarbital $(80 \mathrm{mg} / \mathrm{kg})$. Within 5-6 min of pentobarbital injection, mice were transcardially perfused with PBS followed by $4 \%$ paraformaldehyde (PFA) in PBS.

Mouse brains were dissected and postfixed in 4\% PFA overnight. Brains were cryoprotected in $30 \%$ sucrose for $24 \mathrm{~h}$, frozen in Optimal Cutting Temperature compound (Sakura Finetek), and coronally sectioned at $40 \mu \mathrm{m}$ thickness on a cryostat. Sections were stored in antifreeze solution at $-20^{\circ} \mathrm{C}$ until staining. 

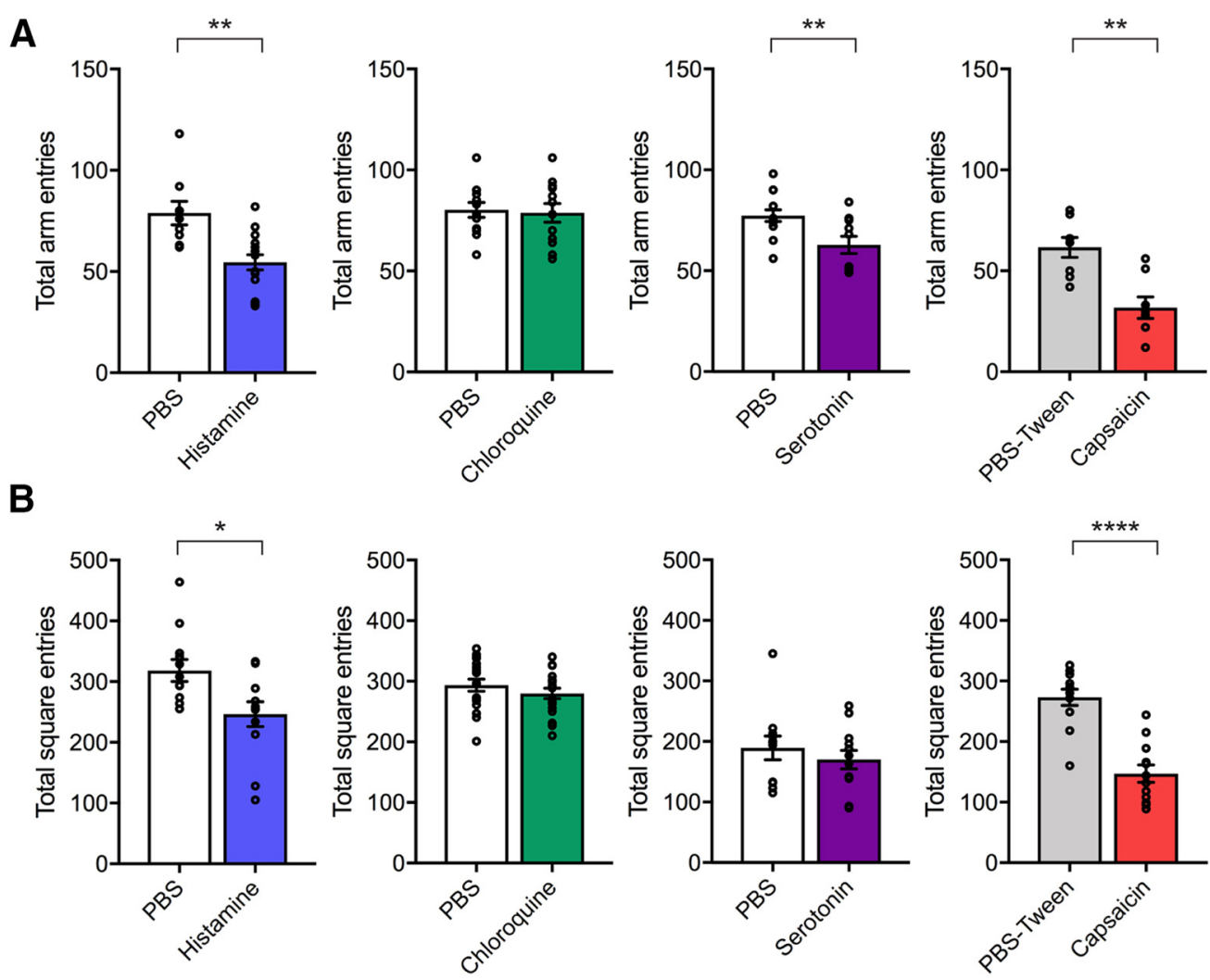

Figure 2. Acute itch stimuli sometimes, but not always, reduce locomotion. $A, 0$ n the EPM, histamine, serotonin, and capsaicin reduced the total number of arm entries, a measure of locomotion.

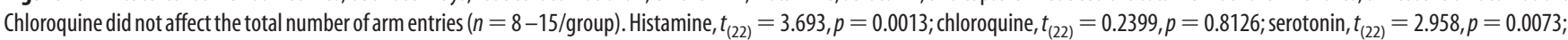
capsaicin, $t_{(14)}=4.122, p=0.0010 . B, 0$ n the OFT, histamine and capsaicin reduced the total number of square entries, a measure of locomotion. Chloroquine and serotonin did not affect the total number of square entries ( $n=11-18 /$ group). Histamine, $t_{(22)}=2.651, p=0.0146$; chloroquine, $t_{(34)}=1.044, p=0.3038$; serotonin, $t_{(21)}=0.782, p=0.4429 ;$ capsaicin, $t_{(22)}=6.429, p<$ 0.0001. Data are shown as mean \pm SEM. ${ }^{*} p<0.05,{ }^{* *} p<0.01,{ }^{* * * *} p<0.0001$ for unpaired $t$ test versus vehicle.

For free-floating immunohistochemistry, sections were washed with PBS and then blocked with 5\% normal donkey serum in PBS with $0.2 \%$ Triton X-100 for $2 \mathrm{~h}$ at room temperature. Sections were incubated with goat-anti-c-Fos primary antibody (1:100; sc-52-G; Santa Cruz Biotechnology; RRID:AB_2629503) in blocking buffer for $48 \mathrm{~h}$ at $4^{\circ} \mathrm{C}$. Following primary incubation, sections were washed with PBS, incubated with donkey-anti-goat secondary antibody conjugated with AlexaFluor 488 (1:300; Invitrogen) in blocking buffer for $2 \mathrm{~h}$ at room temperature, washed again, and mounted on slides with VECTASHIELD Hardset Antifade Mounting Medium with DAPI (Vector Laboratories).

Pilot studies were used to establish the sections with the highest c-Fos activity within the amygdala, $\mathrm{PBN}, \mathrm{MCC}$, and $\mathrm{mPFC}$ regions following itch stimulation. The following anterior-posterior (AP) coordinates were chosen for quantification: amygdala: $-1.22,-1.34$, and $-1.46 \mathrm{~mm}$ from bregma; PBN: -5.02 and $-5.20 \mathrm{~mm}$; MCC: $-0.70 \mathrm{~mm}$; and mPFC: $+1.54 \mathrm{~mm}$. Of note, peak itch-evoked c-Fos PBN coordinates were consistent with previously reported results (Mu et al., 2017).

To confirm that the selected areas of the PBN, MCC, and mPFC included direct projections to the amygdala, R26 ${ }^{\mathrm{Ai} 14}$ mice were anesthetized with sodium pentobarbital $(65 \mathrm{mg} / \mathrm{kg})$ and mounted in a stereotaxic frame. A retrograde tracer [rAAV2-Retro/CAG-Cre; UNC Vector Core, Chapel Hill, NC; created by Ed Boyden, Massachusetts Institute of Technology (MIT)] was injected into the left or right amygdala using the following stereotaxic coordinates: AP $-1.34 \mathrm{~mm}$; medial-lateral (ML) $\pm 2.7 \mathrm{~mm}$; dorsal-ventral (DV) $-4.5 \mathrm{~mm}$. The injection volume was $0.25 \mu \mathrm{l}$, injected over $1 \mathrm{~min}$ using a glass needle and plunger, as in (Harris et al., 2012). The viral dose was $5.3 \times 10^{12}$ vector genomes $/ \mathrm{ml}$. After 2 weeks, mice were killed, brains were processed for sectioning, and tdTomato expression was imaged under fluorescence microscopy. Each area of c-Fos quantification (except the superior lateral $\mathrm{PBN}$ ) included robust projections to the amygdala.
Stitched photomicrographs of whole sections were obtained using fluorescence microscopy (Leica Microsystems) at $10 \times$ objective magnification. For quantification, structural boundaries were drawn using ImageJ software with reference to Franklin and Paxinos (2008) and Vogt and Paxinos (2014). The number of c-Fos + neurons in each region of interest was counted manually by a trained observer blinded to the treatment condition.

Electrophysiology. For in vivo single-unit recording from the amygdala, C57BL/6J mice were anesthetized with urethane $(1.5 \mathrm{~g} / \mathrm{kg}$, i.p.) and mounted in a stereotaxic frame. A craniotomy was performed above the amygdala, and a tungsten microelectrode was inserted into the amygdala using the following stereotaxic coordinates: AP $-1.34 \mathrm{~mm}$; $\mathrm{ML}-2.7$ $\mathrm{mm}$; DV $-4.5 \mathrm{~mm}$. Unit activity was amplified and digitally displayed using Powerlab (A-D Instruments) and Spike2 software (CED Instruments). Action potentials were sorted by spike size and waveform and quantified as number of impulses per second.

Histamine-responsive units were isolated by a chemical search strategy, as in (Akiyama et al., 2009a). Briefly, a small (0.1 $\mu \mathrm{l})$ intradermal microinjection of histamine $(50 \mu \mathrm{g} / \mu \mathrm{l})$ was made in the ventral hindpaw, and a unit exhibiting ongoing activity was isolated. After the ongoing activity subsided, $1 \mu$ l of histamine was injected through the same needle. Firing of the unit was recorded in response to the following stimuli to the foot: innocuous brush, noxious pinch, intradermal PBS, chloroquine $(100 \mu \mathrm{g} / 1 \mu \mathrm{l}$, i.d.), serotonin $(10 \mu \mathrm{g} / 1 \mu \mathrm{l}$, i.d.), intradermal PBS-Tween, and capsaicin $(30 \mu \mathrm{g} / 1 \mu \mathrm{l}$, i.d.).

At the conclusion of the experiment, an electrolytic lesion was made at the recording site, and brains were processed for sectioning. Lesions were labeled with Prussian blue reaction for the demonstration of iron (Polysciences) and were identified under light microscopy with reference to Franklin and Paxinos (2008).

TRAP system and AAV injection. To obtain genetic access to histamineor PBS-responsive amygdala neurons, we used the Targeted Recombina- 

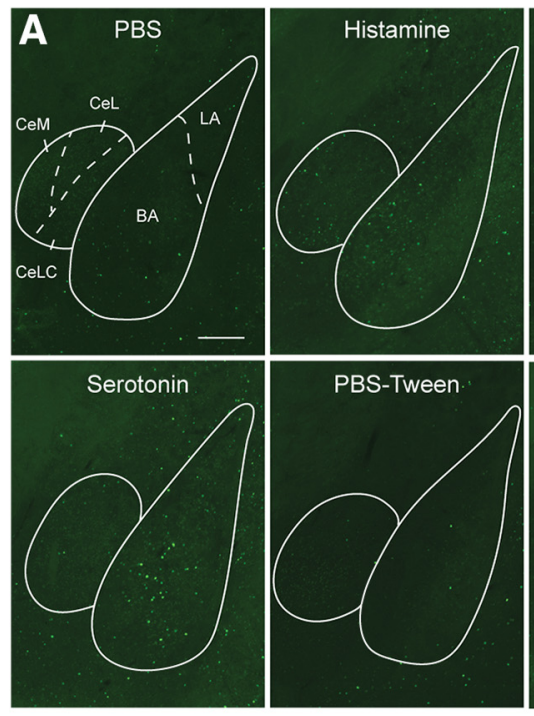

D

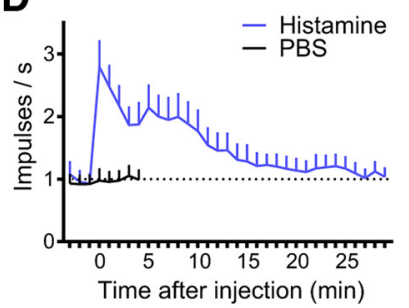

E

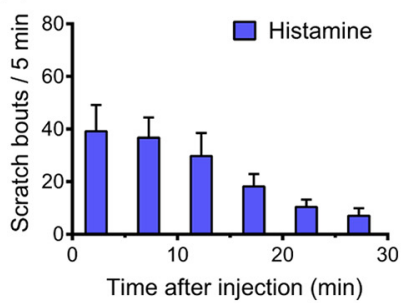

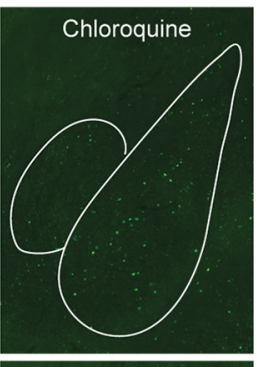

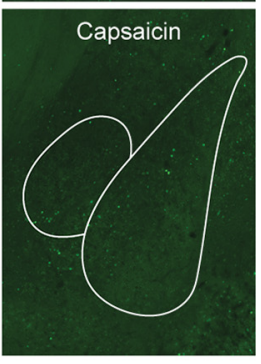

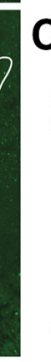
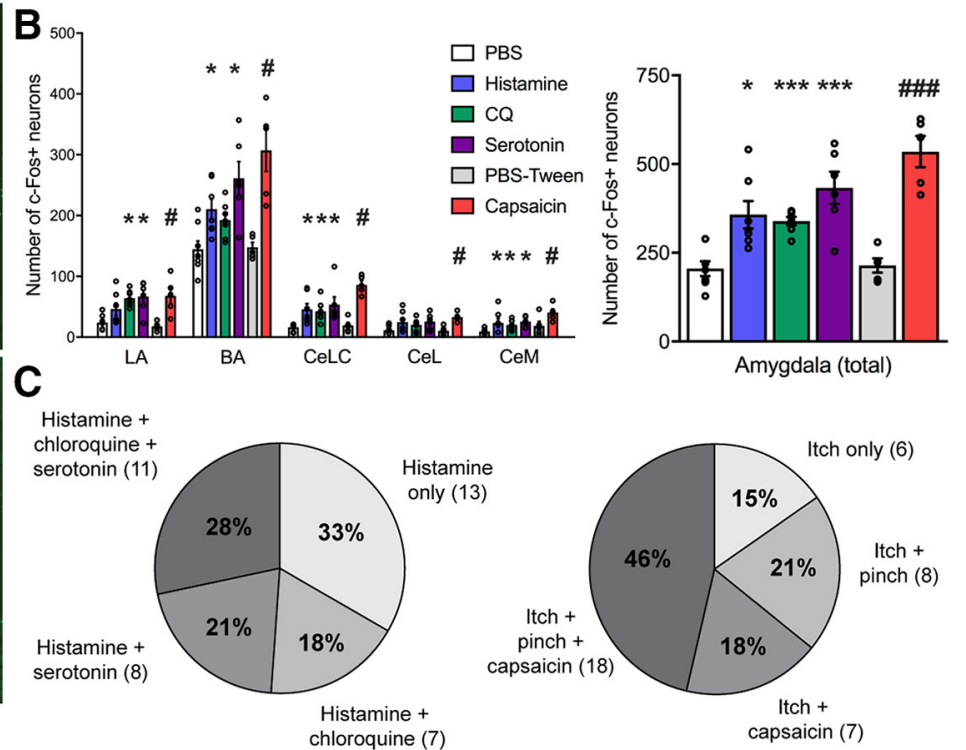

Amygdala (total)
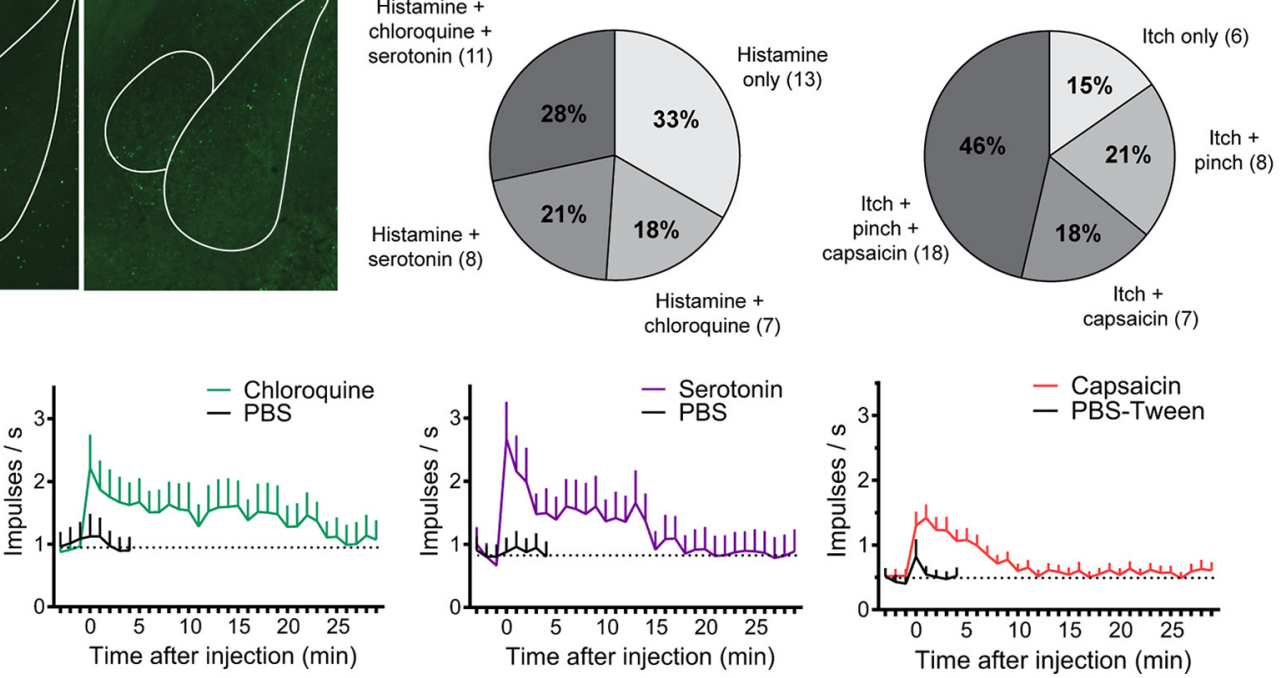

$\mathbf{F}$

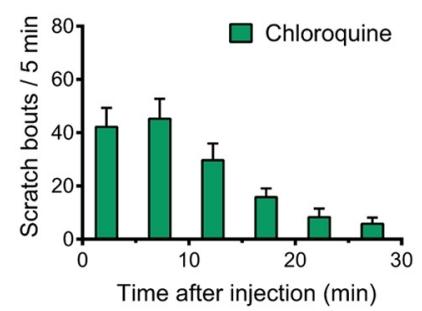

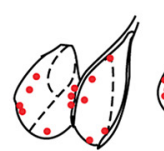

Bregma $-1.06 \mathrm{~mm}$

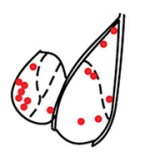

Bregma $-1.34 \mathrm{~mm}$

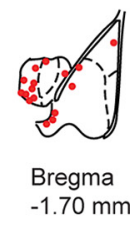

Figure 3. A population of amygdala neurons responds to intradermal injection of histamine and other pruritogens. $A$, Representative images of c-Fos immunostaining (green) in amygdala following intradermal injection of PBS, histamine, chloroquine, serotonin, PBS-Tween, or capsaicin. Scale bar, $250 \mu \mathrm{m}$. LA and BA subdivisions of the BLA and CeLC, CeL, and CeM subdivisions of the CeA are indicated. $\boldsymbol{B}$, Itch and pain stimuli induced significant increases in the number of c-Fos + neurons in the amygdala, including the CeLC region ( $n=5-8 / \mathrm{group})$. Total amygdala: histamine, $t_{(13)}=3.625, p=0.0031$; chloroquine, $t_{(13)}=5.488, p=0.0001$; serotonin, $t_{(12)}=4.983, p=0.0003$; capsaicin, $t_{(8)}=6.625, p=0.0002$. C, In vivo single-unit electrophysiological recording from histamine-responsive amygdala neurons revealed that the majority of neurons also responded to other itch and/or pain stimuli. $D$, Average firing response of responsive units to intradermal histamine $(n=39)$, chloroquine $(n=18)$, serotonin $(n=19)$, and capsaicin $(n=25)$. The firing response of the same units to vehicle injection is shown for comparison. Dotted line indicates average baseline. $\boldsymbol{E}$, Average time course of scratching in awake, behaving mice injected with intradermal histamine, chloroquine, or serotonin. $\boldsymbol{F}$, Electrophysiological recording sites. Data in $\boldsymbol{B}, \boldsymbol{D}$,

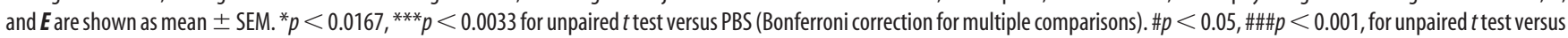
PBS-Tween.

tion in Active Populations (TRAP) system (Guenthner et al., 2013). Fos: $\mathrm{CreER}^{\mathrm{T} 2} ; \mathrm{R} 26^{\mathrm{Ail} 4}$ mice received an i.p. injection of 4-hydroxytamoxifen $(50 \mathrm{mg} / \mathrm{kg}$ in Chen oil; Sigma-Aldrich) followed $10 \mathrm{~min}$ later by an injection of PBS vehicle or histamine $(50 \mu \mathrm{g} / 10 \mu \mathrm{l}$, i.d.). After $\sim 2$ weeks, mice were anesthetized with sodium pentobarbital (65 mg/kg, i.p.) and positioned in a stereotaxic frame. An AAV encoding a Cre-dependent fast opsin (Chronos; Klapoetke et al., 2014) fused to enhanced green fluorescent protein (rAAV5/Syn-FLEX-Chronos-GFP; UNC Vector Core; created by Ed Boyden, MIT) was bilaterally injected into the CeA (coordinates: AP $-1.34 \mathrm{~mm}, \mathrm{ML} \pm 2.7 \mathrm{~mm}, \mathrm{DV}-4.5 \mathrm{~mm}$ ). The injection volume was $0.25 \mu$ l, injected over 1 min using a glass needle and plunger. The viral dose was $3.6 \times 10^{12}$ vector genomes $/ \mathrm{ml}$. An optic fiber (200 $\mu \mathrm{m}$ diameter) was implanted directly above each injection site and fixed to the skull with dental cement. Mice were allowed 3 weeks to recover from surgery before behavior testing.
At the conclusion of behavior testing, mice were perfused as described above. Brains were processed and sectioned and immunostained using a rabbit anti-GFP antibody (1:500; A-11122, Invitrogen; RRID:AB_221569) for $48 \mathrm{~h}$ at $4^{\circ} \mathrm{C}$ followed by a donkey-anti-rabbit secondary antibody conjugated with AlexaFluor 488 (Invitrogen). Fluorescence microscopy was used to confirm colocalization of tdTomato with GFP. Amygdala tdTomato expression was quantified as described above for c-Fos.

Optogenetic stimulation and behavior testing. For optic stimulation, double flexible fiber patch cords were attached to the external ends of the optic fibers and connected to an LED light source (Prizmatix) that delivered blue light (460 $\mathrm{nm}$ wavelength) capable of activating Chronos. Light pulses were delivered at constant intensity and frequency ( 3 $\mathrm{mW}, 20 \mathrm{~Hz}$ ) via an LED driver connected to a waveform generator. 
A
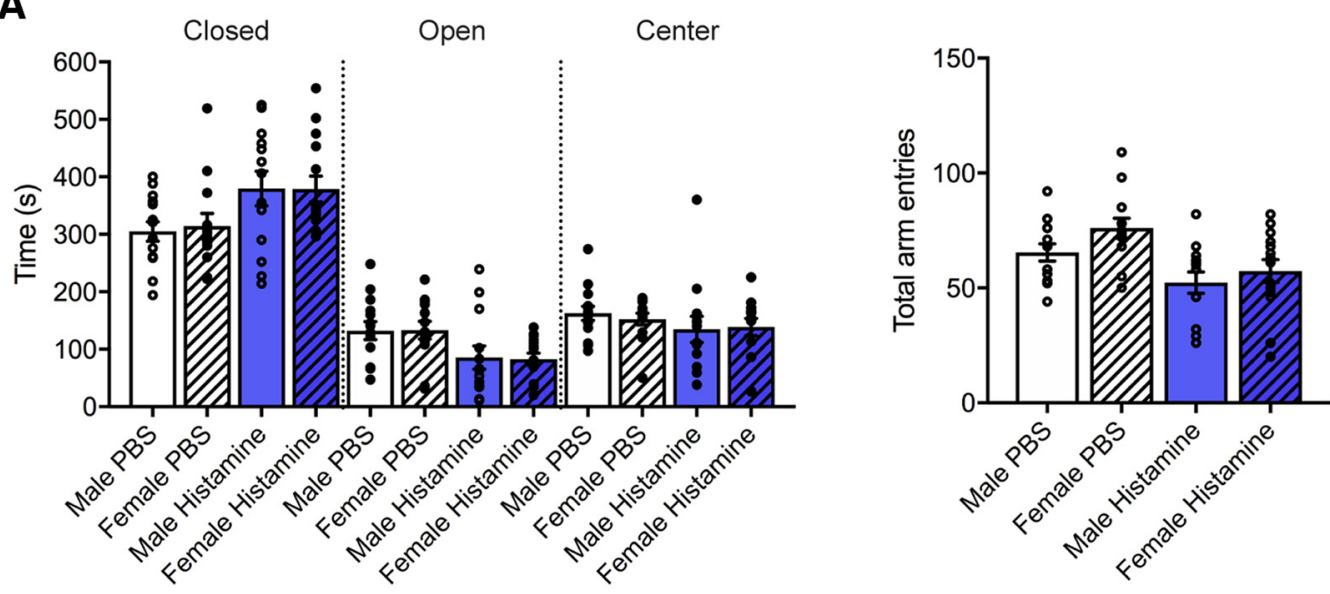

B
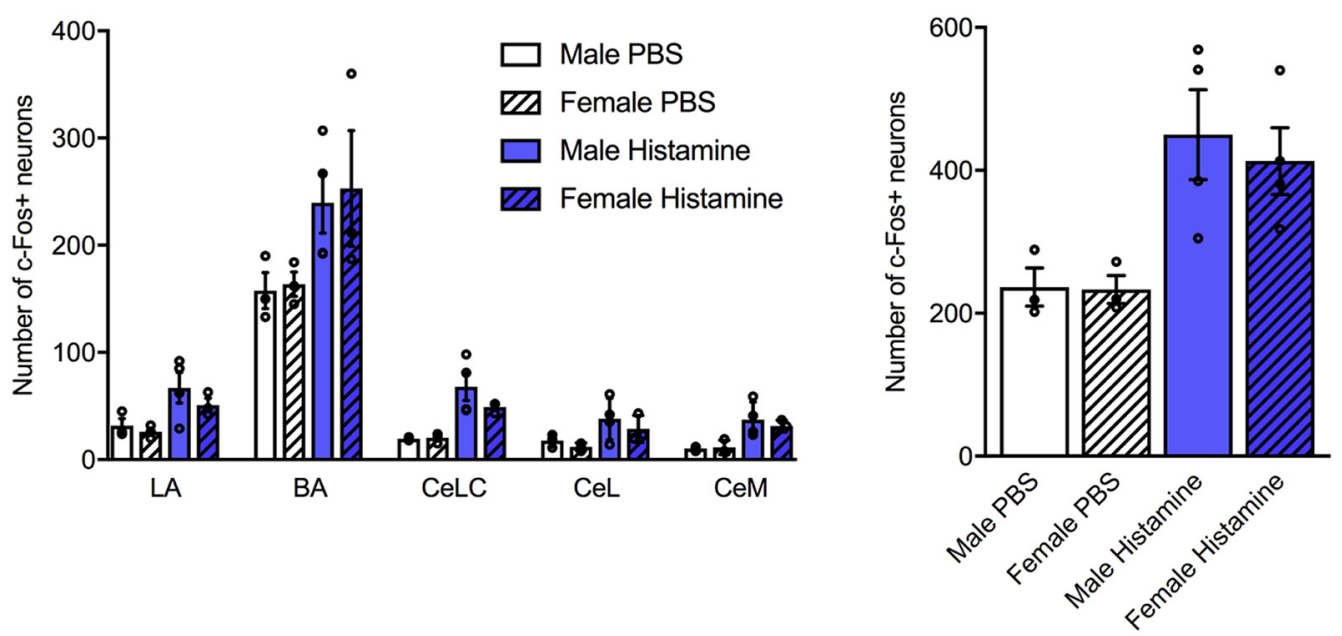

Figure 4. Sex differences in itch-evoked anxiety were not observed. $A$, Male and female mice displayed similar closed arm time, open arm time, center time, and total arm entries on the EPM following PBS injection as well as following histamine injection ( $n=13-14 /$ group). Open arm time: interaction, $F_{(1,50)}=0.01671, p=0.8977 ;$ treatment, $F_{(1,50)}=9.622, p=0.0032 ;$ sex, $F_{(1,50)}$ $=0.0047, p=0.9456$. Total entries: interaction, $F_{(1,50)}=0.3921$; treatment, $F_{(1,50)}=12.97, p=0.0007 ;$ sex, $F_{(1,50)}=3.138, p=0.0826$. $\boldsymbol{B}$, Male and female mice displayed similar numbers of c-Fos + amygdala neurons following PBS injection as well as following histamine injection $\left(n=3-4 /\right.$ group). Total c-Fos + amygdala neurons: interaction, $F_{(1,9)}=0.06715, p=0.8014$; treatment, $F_{(1,9)}=4.368, p=0.0662 ;$ sex, $F_{(1,9)}=0.09686, p=0.727$. Data are shown as mean \pm SEM. No main effect of sex or interaction effect of sex and treatment, two-way ANOVA.

Light intensity was measured in each fiber using an optical power meter (Thorlabs).

To confirm Chronos function, electrophysiology was used in combination with optic stimulation (Gradinaru et al., 2007). Following TRAPing of histamine-responsive neurons and AAV injection, single-unit recording in the CeA was performed as before. To illuminate the recorded neurons, the tungsten electrode was glued to an optical fiber with $\sim 0.5 \mathrm{~mm}$ offset ( $200 \mu \mathrm{m}$ outer diameter; Thorlabs). The other end of the optical fiber was connected to an LED driver. The electrode was advanced until a unit exhibiting ongoing firing was isolated with blue light illumination. When an active unit was isolated, the blue light was turned off. Then, unit activity was recorded at baseline, during blue light stimulation ( $460 \mathrm{~nm}, 3 \mathrm{~mW}, 20 \mathrm{~Hz}, 5 \mathrm{~s})$, and during red light stimulation $(625 \mathrm{~nm}, 3$ $\mathrm{mW}, 20 \mathrm{~Hz}, 5 \mathrm{~s}$ ) of the recording site.

For behavior testing, mice were briefly anesthetized with isoflurane (3\%) to connect implanted optic fibers to the patch cords. Mice were habituated to cable attachment for $1 \mathrm{~h}$ before testing. Mice were tested on the EPM and OFT, with recording divided into three 3 min epochs (OFFON-OFF or OFF-OFF-OFF) as by Felix-Ortiz et al. (2016). Scratching behavior was recorded for $30 \mathrm{~min}$ following intradermal histamine and chloroquine injection to the rostral back, with ("ON") and without ("OFF") continuous bilateral blue light stimulation. We confirmed that post-tamoxifen, postsurgery PBS-TRAPed and histamine-TRAPed mice displayed normal scratching behavior. However, we observed that the weight and/or stress of the cable attachment for optogenetic stimulation suppressed the scratch response. Importantly, the cable was still attached in the OFF condition to allow us to control for this effect.

Experimental design and statistical analysis. Statistical details of experiments can be found in the figure legends. For scratching, EPM, OFT, and c-Fos/tdTomato quantification, comparisons were made between each treatment group and corresponding vehicle group within the same experiment (two-tailed, unpaired $t$ test). A repeated measures $t$ test was used to analyze CPA outcomes. To assess sex differences in EPM and c-Fos quantification, a two-way ANOVA (sex $\times$ treatment) was used. To assess effects of optogenetic stimulation, a paired $t$ test was used (blue light vs no light). To test for a relationship between scratching and locomotion on EPM and OFT, a Pearson's correlation was used.

In electrophysiology experiments, units were considered responsive if they exhibited a $>3$ SD increase (positive response) or decrease (negative response) in firing rate compared with prestimulus baseline. The mean response duration was calculated by determining the time point in a $60 \mathrm{~s}$ bin when the firing rate dropped below $>3$ SD above prestimulus baseline for at least $2 \mathrm{~min}$. The mean time from injection for the response to reach maximal discharge rate was calculated by determining the time point in a $60 \mathrm{~s}$ bin when the firing rate peaked.

Statistical significance was set at $p<0.05$, except for analyses with multiple comparisons, where significance was set at $p<0.0167$ (Bonfer- 

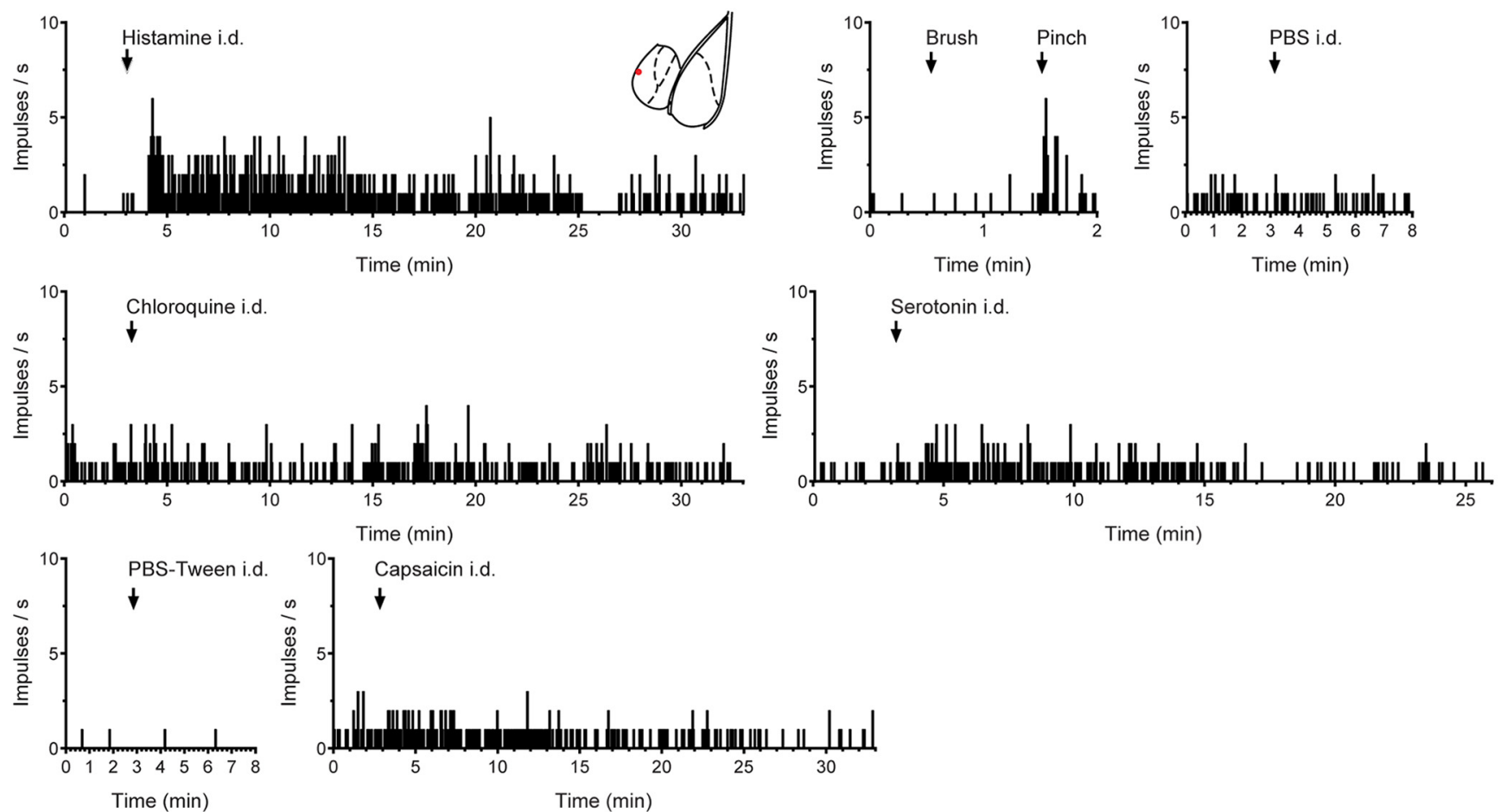

Figure 5. Histamine-responsive amygdala neurons typically respond to multiple itch and/or pain stimuli. Example in vivo single-unit electrophysiological recording from a histamine-responsive unit in the amygdala. This unit displayed a positive response over baseline to intradermal histamine, noxious pinch, and intradermal serotonin. Recording site shown in inset.

roni correction for 3 comparisons). All tests were two-tailed. All statistical analyses and graphs were made using GraphPad Prism 7.

\section{Results}

Acute itch stimuli evoke anxiety-like behavior in mice

To test whether acute, experimentally induced itch evokes anxiety-like behavior in mice, we used intradermal injection of three pruritogens: histamine, chloroquine, and serotonin. These chemicals have been established to induce itch through different peripheral signaling pathways, with chloroquine and serotonin itch being histamine-independent (Liu et al., 2009; Akiyama and Carstens, 2013). At the selected doses, all three pruritogens evoked robust scratching over $30 \mathrm{~min}$ (Fig. 1A). PBS vehicle did not elicit notable scratching. A previous study found that mice display CPA in response to intradermal injection of chloroquine (Mu and Sun, 2017). We confirmed that mice also display avoidance of an itch-associated chamber following intradermal histamine and serotonin (Fig. 1B).

The EPM and OFT measure thigmotaxis: a preference toward contact with walls versus exploring unprotected spaces that is commonly used as a measure of anxiety-like behavior. Mice that received intradermal histamine, chloroquine, or serotonin displayed significantly less open arm time on the EPM compared with mice who received PBS vehicle, indicating that these pruritogens increased thigmotaxis behavior (Fig. 1C). For comparison, intradermal capsaicin, which typically produces pain- but not itch-related behavior (Akiyama et al., 2010), induced a similar reduction in open-arm time compared with its vehicle, PBSTween. Additionally, following histamine, chloroquine, and capsaicin injection, mice displayed a reduction in the percentage of center square entries on the OFT (Fig. 1D). Pruritogeninduced thigmotaxis was sometimes, but not always, accompanied by a reduction in overall locomotion (Fig. 2). The number of scratches displayed on the EPM and OFT was not correlated to
Table 1. Positive response incidences (number/total) of histamine-responsive amygdala neurons to other tested stimuli

\begin{tabular}{lllll}
\hline Brush, \% & Pinch, \% & Chloroquine, \% & Serotonin, \% & Capsaicin, \% \\
\hline $21(8 / 39)$ & $64(25 / 39)$ & $46(18 / 39)$ & $49(19 / 39)$ & $64(25 / 39)$ \\
\hline
\end{tabular}

changes in locomotion $\left(r^{2}=0.018, p=0.3517\right.$ on EPM; $r^{2}=$ $0.000377, p=0.8892$ on OFT).

A population of amygdala neurons responds to intradermal injection of histamine and other pruritogens

To investigate the activation of potential brain regions associated with itch and anxiety, we used immunohistochemistry to label c-Fos, an immediate-early gene that is widely used as a marker of neuronal activity following nociceptive and other stimuli (Coggeshall, 2005). Representative images of c-Fos + neurons in the amygdala following intradermal PBS, histamine, chloroquine, serotonin, PBS-Tween, or capsaicin injection are shown in Figure $3 A$. Pruritogen injection led to an increased number of c-Fos + neurons in the amygdala (Fig. $3 B$ ). This increase was significant for all four chemicals in the laterocapsular subdivision (CeLC) of the CeA, which has been termed the "nociceptive amygdala" for its major role in pain-related anxiety (Neugebauer, 2015). We did not observe sex differences in anxiety-like behavior (Fig. 4A) or amygdalar c-Fos expression (Fig. 4B) following either PBS or histamine injection.

To functionally characterize the population of itch-responsive neurons in the amygdala, we used in vivo single-unit electrophysiological recording. Histamine-responsive neurons were isolated using a chemical search strategy, and their firing rates were measured in response to various somatosensory stimuli. Recording from an example unit is shown in Figure 5. Response incidences of histamine-responsive neurons to other tested stimuli are listed in Table 1. A majority of histamine-responsive neurons were also 
A

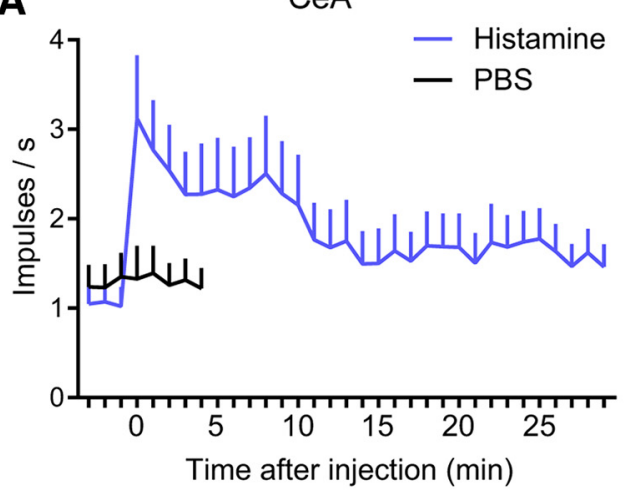

B

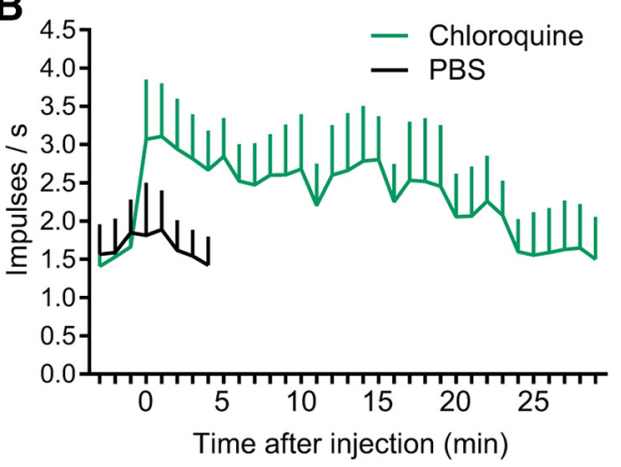

C

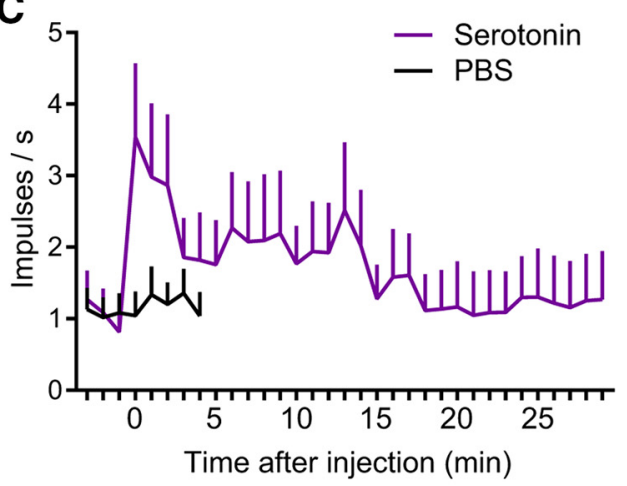

D

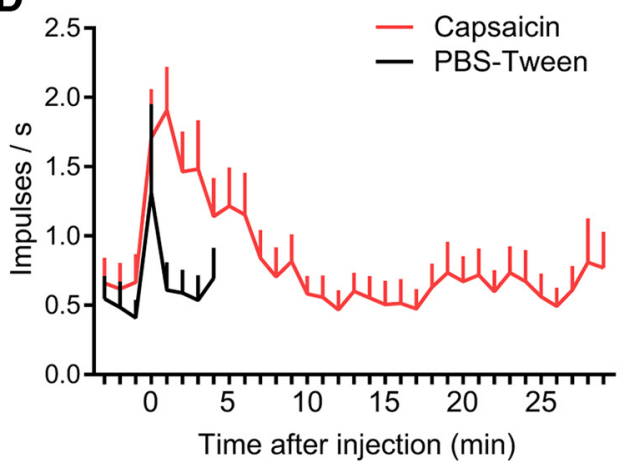

BLA
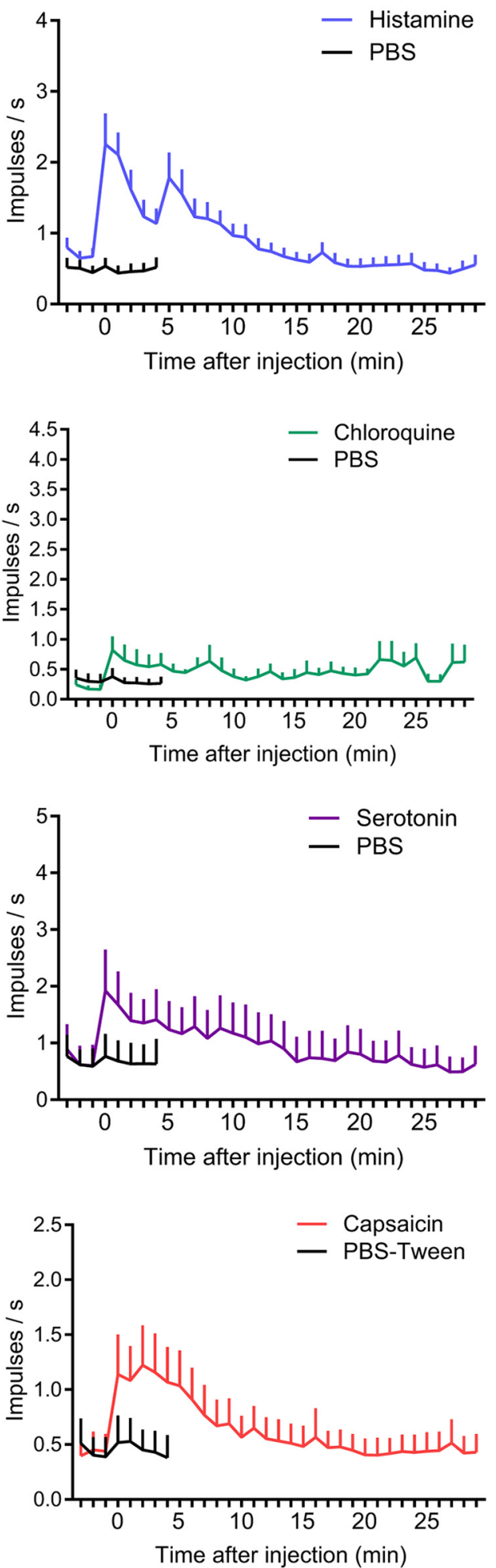

Figure 6. Pruritogen-evoked firing tended to be of greater magnitude and duration in the CeA compared with the BLA. $\boldsymbol{A}$, Average firing of units recorded in the CeA or BLA that positively responded to intradermal histamine. $\boldsymbol{B}$, As in $\boldsymbol{A}$, for intradermal chloroquine. $\boldsymbol{C}$, As in $\boldsymbol{A}$, for intradermal serotonin. $\boldsymbol{D}$, As in $\boldsymbol{A}$, for intradermal capsaicin. Data are shown as mean \pm SEM.

excited by non-histaminergic pruritogens and/or by painful stimuli (Fig. 3C).

Figure $3 D$ shows the average firing response of histamineresponsive amygdala neurons to intradermal histamine, chloroquine, serotonin, and capsaicin. For each pruritogen, the time course of neuronal firing responses was behaviorally relevant, roughly matching the time course of scratching behavior in awake, behaving mice (Fig. $3 E$ ). The average firing response to each pruritogen was of greater magnitude and duration for units recorded in the CeA compared with those recorded in the BLA (Fig. 6). Recording sites are shown in Figure $3 F$, as determined by histologic examination of postrecording electrolytic lesions. 
A
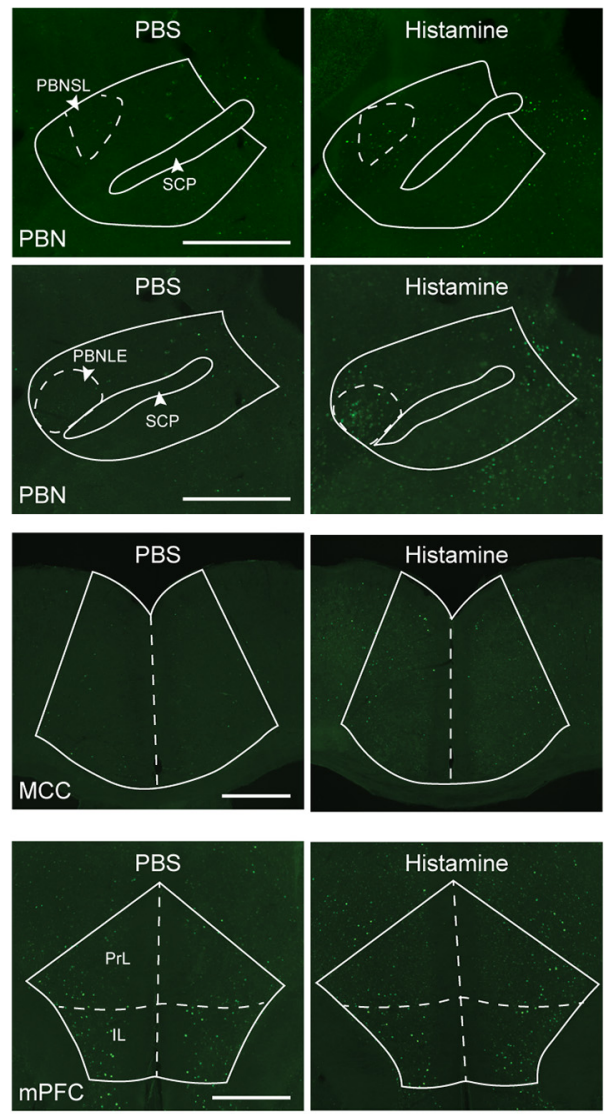

B

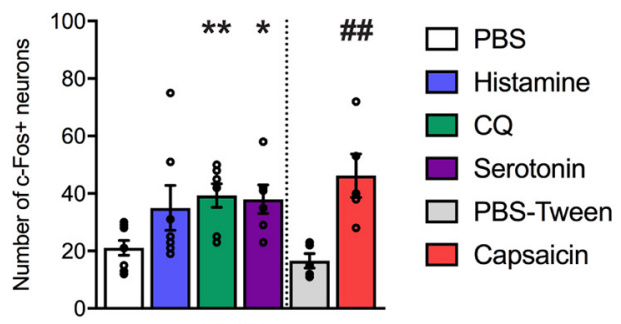

PBNSL

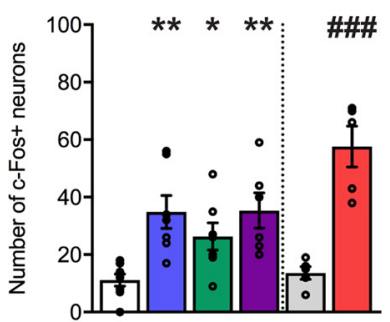

PBNLE
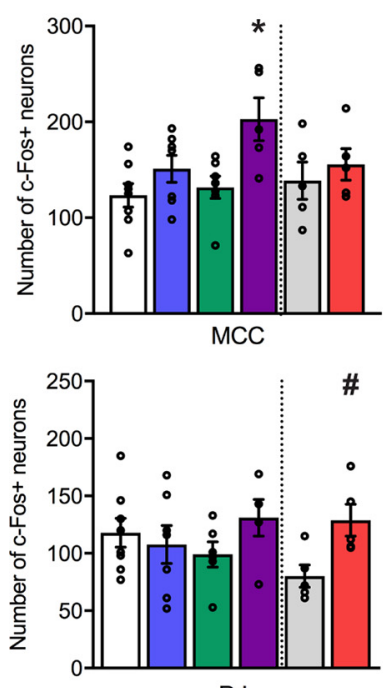

PrL

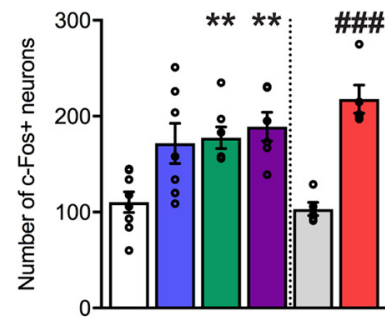

PBN (total)

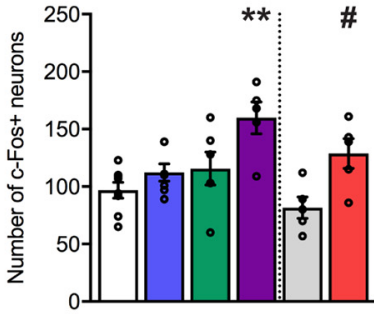

IL

Figure 7. Acute itch is associated with increased neuronal activity in the PBN, MCC, and $\mathrm{mPFC}$, projection areas to the amygdala. A, Representative images of c-Fos immunostaining in the PBN, MCC, and mPFC following intradermal injection of PBS or histamine. Superior lateral subdivision (PBNSL), PBNLE, and superior cerebellar peduncle (SCP) are indicated for PBN. PrL and IL subdivisions are indicated for mPFC. Scale bar, $500 \mu \mathrm{m}$. B, Quantification of c-Fos + neurons in PBN, MCC, and mPFC following intradermal PBS, histamine, chloroquine, serotonin, PBS-Tween, and capsaicin. PBNSL: histamine, $t_{(13)}=1.782, p=0.0981$; chloroquine, $t_{(13)}=3.846, p=0.0020$; serotonin, $t_{(12)}=3.242, p=0.0071$; capsaicin, $t_{(8)}=3.709, p=0.0060$. PBNLE: histamine, $t_{(13)}=4.106$, $p=0.0012$; chloroquine, $t_{(13)}=3.069, p=0.0090$; serotonin, $t_{(12)}=4.186, p=0.0013$; capsaicin, $t_{(8)}=5.937, p=0.0003$. Total PBN: histamine, $t_{(13)}=2.697, p=0.0183 ;$ chloroquine, $t_{(13)}$ $=4.283, p=0.0009$; serotonin, $t_{(12)}=4.388, p=0.0009$; capsaicin, $t_{(8)}=7.078, p=0.0001$. MCC: histamine, $t_{(13)}=1.489, p=0.1603 ;$ chloroquine, $t_{(13)}=0.4919, p=0.6310 ;$ serotonin, $t_{(11)}=3.405, p=0.0059$; capsaicin, $t_{(8)}=0.6635, p=0.5256$. PrL: histamine, $t_{(13)}=0.4968, p=0.6276$; chloroquine, $t_{(12)}=1.085, p=0.2993 ;$ serotonin, $t_{(11)}=0.647, p=0.5309 ;$ capsaicin, $t_{(8)}=2.865, p=0.0210$. IL: histamine, $t_{(13)}=1.529, p=0.1503$; chloroquine, $t_{(12)}=1.293, p=0.2203$; serotonin, $t_{(11)}=4.535, p=0.0009 ;$ capsaicin, $t_{(8)}=2.96, p=0.0182$. Data in $B$ are shown as mean \pm SEM. ${ }^{*} p<0.0167,{ }^{* *} p<0.0033$ for unpaired $t$ test versus PBS (Bonferroni correction for multiple comparisons). $\# p<0.05$, \#\#p $<0.01$, \#\#\#p $<0.001$ for unpaired $t$ test versus PBS-Tween.

We additionally investigated itch-evoked activity in the PBN, MCC, and mPFC. Representative images of c-Fos staining in the PBN, MCC, and mPFC are shown in Figure 7A. Chloroquine, serotonin, and capsaicin induced a significant increase in the number of c-Fos+ neurons in the PBN (Fig. 7B). Within the $\mathrm{PBN}$, the lateral external subdivision (PBNLE), which is known to project to the CeLC ( $\mathrm{Lu}$ et al., 2015), was significantly activated by all four tested chemicals. Additionally, we found that chloroquine, serotonin, and capsaicin increased activity in the superior lateral subdivision of the PBN. This area includes few projections to the amygdala but has strong connections with the hypothalamus (Bester et al., 1997). Therefore, this represents a potential pathway for itch-related stress that does not directly involve the amygdala.
Serotonin induced activation of the MCC, suggesting that this area may modulate itch-evoked BLA activity. Finally, capsaicin was associated with activation in both the prelimbic (PrL) and infralimbic (IL) divisions of the MPFC, but serotonin was associated with activation only in the IL division. Therefore, the mPFC may not play a major role in moderating anxiety from acute itch stimuli; the function of this region in the condition of chronic itch remains to be investigated.

Optogenetic stimulation of histamine-responsive amygdala neurons affects itch- and anxiety-related behavior

To directly tag and manipulate histamine-signaling neurons in the amygdala, we used the TRAP system, which allows genetic access to physiologically classified neurons. A schematic diagram 
A

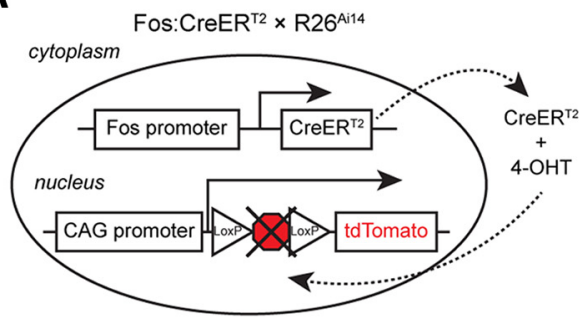

TRAP stimulus: i.d. PBS or histamine

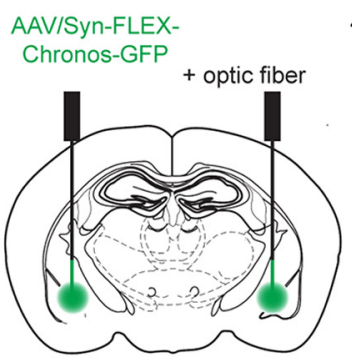

$460 \mathrm{~nm}$ light stimulation

ON/OFF

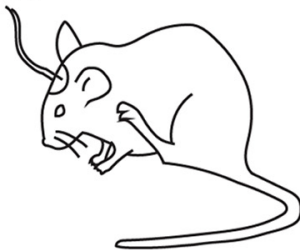

Anxiety and itch

behavior testing
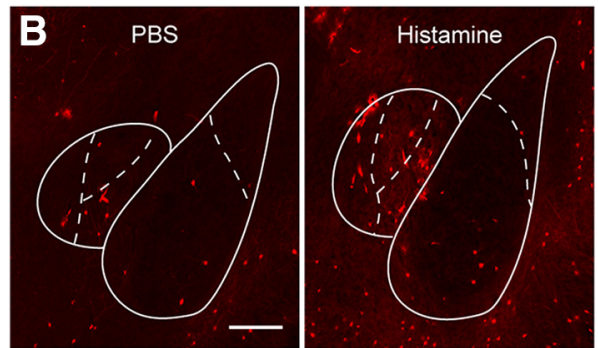

D

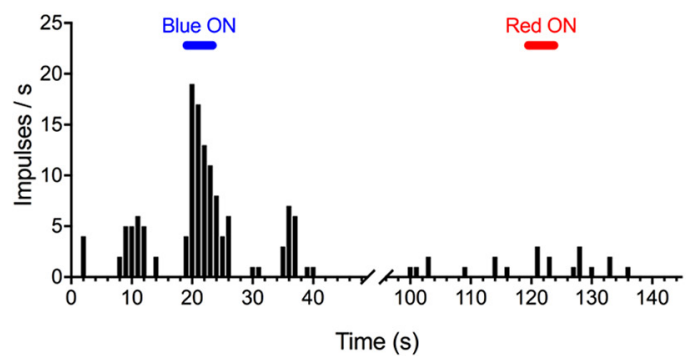

$\mathbf{F}$
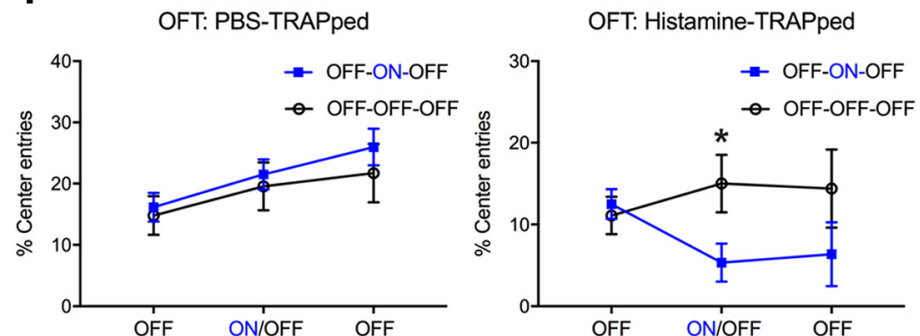
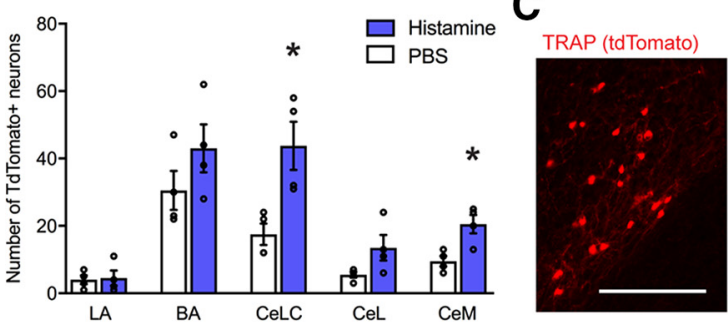
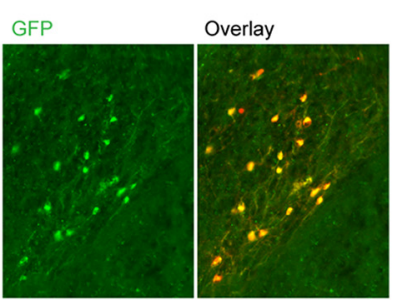

E

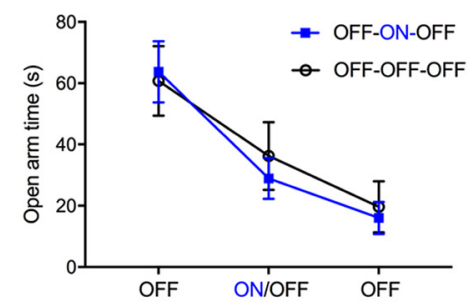

EPM: Histamine-TRAPped

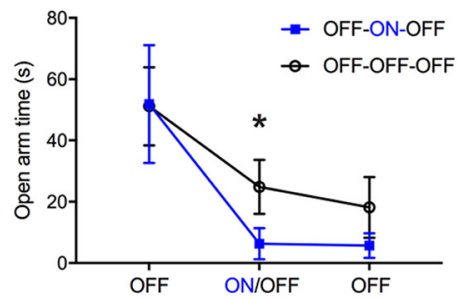

G

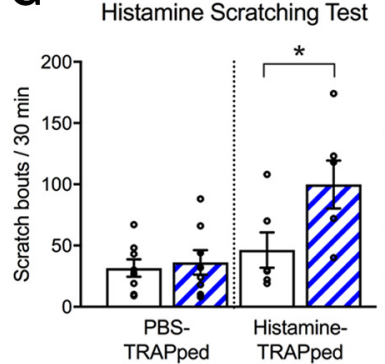

Chloroquine Scratching Test

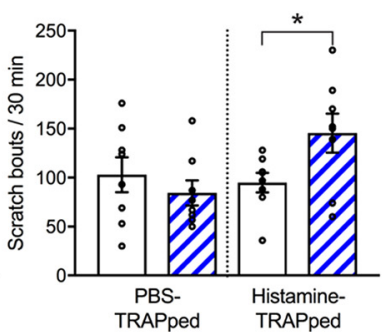

Figure 8. Optogenetic stimulation of histamine-responsive amygdala neurons affects itch- and anxiety-related behavior. $\boldsymbol{A}$, Schematic of experimental procedure. $\boldsymbol{B}$, In TRAP mice, intradermal histamine injection resulted in significantly more tdTomato $+\left(\right.$ red) amygdala neurons than intradermal PBS $\left(n=4 /\right.$ group). Scale bar, $250 \mu \mathrm{m} . \mathrm{LA}: t_{(6)}=0.1925, p=0.8537 ; \mathrm{BA}: t_{(6)}=1.36, p=$ 0.2226 ; CeLC: $t_{(6)}=3.362, p=0.0152 ; C_{\text {Cl: }} t_{(6)}=2.054, p=0.0857 ;$ CeM: $t_{(6)}=3.508, p=0.0127$. C, Representative images of AAV-transfected histamine-TRAPed cells in the CeA. Scale bar, $250 \mu \mathrm{m}$. D, Example in vivo electrophysiological recording of a TRAPed amygdala neuron. Under $20 \mathrm{~Hz}$ blue light stimulation, but not red light stimulation, the TRAPed cell displayed an increased firing rate. $\boldsymbol{E}, 0$ n the $E P M$, blue light stimulation significantly reduced open arm time for histamine-TRAPed mice. PBS-TRAPed ON versus 0 FF, $t_{(6)}=0.7049, p=0.5036$; histamine-TRAPed $0 \mathrm{~N}$ versus OFF, $t_{(6)}=2.448, p=0.0499$. $\boldsymbol{F}$, As in $\boldsymbol{E}$, for percentage center entries on OFT. PBS-TRAPed ON versus OFF, $t_{(6)}=0.6082, p=0.5654$; histamine-TRAPed ON versus OFF, $t_{(6)}=2.802, p=$ 0.0311. G, Blue light stimulation greatly enhanced the total scratch response of histamine-TRAPed mice (but not PBS-TRAPed mice) to histamine and chloroquine over 30 min. Histamine scratching: PBS TRAPed ON versus OFF, $t_{(7)}=0.2847, p=0.7841$; histamine-TRAPed ON versus OFF, $t_{(5)}=2.656, p=0.0451$. Chloroquine scratching: PBS TRAPed ON versus OFF, $t_{(7)}=0.8756, p=0.4103$; histamine-TRAPed ON versus OFF, $t_{(7)}=3.092, p=0.0175$. Data in $\boldsymbol{B}$ and $\boldsymbol{E}-\boldsymbol{G}$ are shown as mean \pm SEM. ${ }^{*} p<0.05$, for unpaired $t$ test vs PBS $(\boldsymbol{B})$. ${ }^{*} p<0.05$, for paired $t$ test $(\boldsymbol{E}-\boldsymbol{G})$.

of this experiment is shown in Figure $8 A$. Briefly, Fos:CreER ${ }^{\mathrm{T} 2}$; R26 ${ }^{\text {Ail } 14}$ mice were injected with i.p. 4-hydroxytamoxifen to effect Cre recombination and tdTomato expression in active (Fosexpressing) neurons following either intradermal histamine or PBS control. Mice that received PBS displayed few tdTomatopositive cells ("PBS-TRAPed neurons") in the amygdala, while mice that received histamine had many tdTomato-positive cells ("histamine-TRAPed neurons"; Fig. 8B). The number of histamine-TRAPed neurons was significantly greater than the number of PBS-TRAPed neurons in the CeLC and medial CeA (CeM) subdivisions.

On average, the number of PBS- or histamine-TRAPed neurons was approximately similar to the number of c-Fos + neurons in the CeA but was reduced in the BLA. A previous study (Xiu et al., 2014) found that different stimulus timing was necessary to induce maximal c-Fos expression in the CeA versus the BLA. This, in combination with tamoxifen concentration kinetics, likely led to less TRAPing of the BLA. 
To place PBS- and histamine-TRAPed neurons under optogenetic control, mice received bilateral intra-amygdalar injections of AAV/Syn-FLEX-Chronos-GFP, which induces expression of Chronos (a fast opsin that excites neurons under blue light stimulation) and GFP marker in a Cre-dependent manner. Optic fibers were implanted directly above the injection sites. GFP was expressed in $87 \%$ of neurons with tdTomato, indicating successful transfection. Representative images of tdTomato, GFP, and overlay within the CeA are shown in Figure $8 C$. As expected, an amygdala neuron expressing Chronos displayed increased activity under blue light but not red light (Fig. 8D). (Electrophysiological recording during light stimulation was obtained from a single neuron as a proof of concept.)

Behavior testing was conducted with and without blue light stimulation of the amygdala to assess the impact of the TRAPed neuronal populations. On the EPM, mice with PBS-TRAPed neurons did not display any change in behavior with optic stimulation (Fig. 8E). However, mice with histamine-TRAPed neurons displayed a significant reduction in open arm time during optic stimulation. Similarly, in the OFT, mice with PBS-TRAPed neurons did not respond to optic stimulation, but mice with histamine-TRAPed neurons showed a reduction in percentage center entries during optic stimulation (Fig. 8F).

Finally, we measured scratching response with and without continuous $20 \mathrm{~Hz}$ blue light stimulation of TRAPed neurons. We did not observe spontaneous scratching during light stimulation of either PBS- or histamine-TRAPed amygdala neurons. However, in mice with histamine-TRAPed neurons, optic stimulation resulted in an increased total scratching response to intradermal injection of histamine or chloroquine (Fig. 8G). Mice with PBSTRAPed neurons did not show any change in scratching response during optic stimulation. Therefore, optogenetic stimulation of histamine-responsive amygdala neurons was sufficient to enhance both thigmotaxis and scratching behavior.

\section{Discussion}

Recent studies suggest that many types of sensory stimuli activate specialized neural ensembles in the amygdala and other limbic areas that encode positive or negative emotional valence (Xiu et al., 2014; Kim et al., 2016, 2017). This emotional valence can in turn prompt specific behaviors: for example, avoiding an area where pain was previously experienced. Our results strongly support the hypothesis that itch is an aversive stimulus which activates a population of amygdala neurons to promote emotionally driven responses: avoidance (measured in the CPA test), thigmotaxis (measured in the EPM and OFT), and scratching.

Itch signals, like pain signals, are transmitted to the brain through two major pathways: the spino-thalamic pathway and the spino-parabrachial pathway (Davidson et al., 2012; Akiyama et al., 2015; Jansen and Giesler, 2015; Mu et al., 2017). In pain research, it has been suggested that the spino-parabrachial pathway is critical for conveying affective/motivational information (Han et al., 2015). Within the PBNLE, calcitonin gene-related peptide (CGRP)-expressing neurons are activated by many types of threat stimuli and project this information to the CeLC. Inactivation of this pathway attenuated footshock-induced fear conditioning without affecting pain withdrawal latency (Han et al., 2015). We observed that histamine, chloroquine, and serotonin all induced significant activation of the PBNLE. It has also been reported that silencing CGRP $+\mathrm{PBN}$ neurons reduced scratching following chloroquine injection (Campos et al., 2018). Additionally, blocking glutamatergic transmission in the $\mathrm{PBN}$ suppressed the scratch response to multiple pruritogens and reduced spon- taneous scratching in a mouse model of chronic itch (Mu et al., 2017). Together, these findings suggest that the PBNLE-CeLC pathway is likely to be important for mediating affective itch and scratching behavior.

We found that all three pruritogens induced significant activation of the amygdala. Within the CeA, histamine, chloroquine, and serotonin (as well as capsaicin) induced significant activation of the CeLC. This region is well known for its role in mediating the affective component of pain (Neugebauer, 2015). CeLC neurons expressing the transcription factor Prkcd are activated by many types of threatening or aversive stimuli, such as footshock or bitter quinine water, and drive defensive behavioral responses (Kim et al., 2017). Surprisingly, all three pruritogens also induced activation of the CeM, which is more associated with appetitive stimuli and behaviors (Xiu et al., 2014; Kim et al., 2017). This activation may potentially be related to the rewarding behavior of scratching.

Pruritogens also induced elevations of activity in the BLA. Our area of c-Fos quantification was located within the anterior BLA, in which neurons expressing the transcription factor Rspo 2 predominate (Kim et al., 2016). This population was found to be activated by aversive odor, taste, and pain (footshock) stimuli. In particular, chloroquine and serotonin evoked significant activity in the lateral (LA) subdivision. This region is known to receive sensory information via the spino-thalamic pathway and cortical areas to establish a conditioned fear response (Ehrlich et al., 2009). Synaptic plasticity of itch-signaling neurons in the LA may therefore contribute to itch-evoked CPA.

The majority of histamine-responsive amygdala neurons were also activated by non-histaminergic itch and/or pain stimuli. Previous studies have found that, although amygdala neurons do not typically respond to both appetitive and aversive stimuli, they are commonly activated by multiple stimuli of the same valence (e.g., by both morphine and cocaine; Xiu et al., 2014). Therefore, the overlap between itch and pain signaling may represent a convergence of information into a more general "aversion" signal. At the same time, a minority of neurons responded to itch only and not to pain. Considering the heterogeneity of amygdala neurons (Kim et al., 2017), we speculate that neurons that respond to many aversive stimuli may promote general anxiety-related behaviors (such as avoidance or thigmotaxis), whereas neurons that preferentially respond to itch may promote itch-specific behavior (such as scratching).

Chronic itch is a significant medical and socioeconomic problem with few available treatments. However, methods that reduce anxiety, such as the use of selective serotonin reuptake inhibitors and psychotherapy, have shown some success in reducing chronic itch. Identifying the projections and neurotransmitters that specifically modulate affective itch will offer a path forward toward developing new treatments that break the itch-anxiety cycle.

\section{References}

Akiyama T, Carstens E (2013) Neural processing of itch. Neuroscience 250: 697-714.

Akiyama T, Merrill AW, Carstens MI, Carstens E (2009a) Activation of superficial dorsal horn neurons in the mouse by a PAR-2 agonist and 5-HT: potential role in itch. J Neurosci 29:6691-6699.

Akiyama T, Merrill AW, Zanotto K, Carstens MI, Carstens E (2009b) Scratching behavior and fos expression in superficial dorsal horn elicited by protease-activated receptor agonists and other itch mediators in mice. J Pharmacol Exp Ther 329:945-951.

Akiyama T, Carstens MI, Carstens E (2010) Differential itch- and painrelated behavioral responses and micro-opioid modulation in mice. Acta Derm Venereol 90:575-581. 
Akiyama T, Nguyen T, Curtis E, Nishida K, Devireddy J, Delahanty J, Carstens MI, Carstens E (2015) A central role for spinal dorsal horn neurons that express neurokinin-1 receptors in chronic itch. Pain 156:1240-1246.

Bester H, Besson JM, Bernard JF (1997) Organization of efferent projections from the parabrachial area to the hypothalamus: a phaseolus vulgarisleucoagglutinin study in the rat. J Comp Neurol 383:245-281.

Campos CA, Bowen AJ, Roman CW, Palmiter RD (2018) Encoding of danger by parabrachial CGRP neurons. Nature 555:617-622.

Chen L, Wang W, Tan T, Han H, Dong Z (2016) GABA receptors in the central nucleus of the amygdala are involved in pain- and itch-related responses. J Pain 17:181-189.

Cho JH, Deisseroth K, Bolshakov VY (2013) Synaptic encoding of fear extinction in mPFC-amygdala circuits. Neuron 80:1491-1507.

Coggeshall RE (2005) Fos, nociception and the dorsal horn. Prog Neurobiol 77:299-352.

Davidson S, Zhang X, Khasabov SG, Moser HR, Honda CN, Simone DA, Giesler GJ Jr (2012) Pruriceptive spinothalamic tract neurons: physiological properties and projection targets in the primate. J Neurophysiol 108:1711-1723.

Duvarci S, Pare D (2014) Amygdala microcircuits controlling learned fear. Neuron 82:966-980.

Ehrlich I, Humeau Y, Grenier F, Ciocchi S, Herry C, Lüthi A (2009) Amygdala inhibitory circuits and the control of fear memory. Neuron 62:757-771.

Felix-Ortiz AC, Burgos-Robles A, Bhagat ND, Leppla CA, Tye KM (2016) Bidirectional modulation of anxiety-related and social behaviors by amygdala projections to the medial prefrontal cortex. Neuroscience 321:197-209.

Ferm I, Sterner M, Wallengren J (2010) Somatic and psychiatric comorbidity in patients with chronic pruritus. Acta Derm Venereol 90:395-400.

Franklin KB, Paxinos G (2008) The mouse brain in stereotaxic coordinates, Ed 3. San Diego: Academic.

Gao YJ, Ji RR (2009) c-fos and pERK, which is a better marker for neuronal activation and central sensitization after noxious stimulation and tissue injury? Open Pain J 2:11-17.

Gradinaru V, Thompson KR, Zhang F, Mogri M, Kay K, Schneider MB, Deisseroth K (2007) Targeting and readout strategies for fast optical neural control in vitro and in vivo. J Neurosci 27:14231-14238.

Guenthner CJ, Miyamichi K, Yang HH, Heller HC, Luo L (2013) Permanent genetic access to transiently active neurons via TRAP: targeted recombination in active populations. Neuron 78:773-784.

Han S, Soleiman MT, Soden ME, Zweifel LS, Palmiter RD (2015) Elucidating an affective pain circuit that creates a threat memory. Cell 162:363374.

Harris JA, Oh SW, Zeng H (2012) Adeno-associated viral vectors for anterograde axonal tracing with fluorescent proteins in nontransgenic and Cre driver mice. Curr Protoc Neurosci Chapter 1:Unit 1.20.1-18.

Jansen NA, Giesler GJ Jr (2015) Response characteristics of pruriceptive and nociceptive trigeminoparabrachial tract neurons in the rat. J Neurophysiol 113:58-70.

Kim J, Pignatelli M, Xu S, Itohara S, Tonegawa S (2016) Antagonistic negative and positive neurons of the basolateral amygdala. Nat Neurosci 19:1636-1646.

Kim J, Zhang X, Muralidhar S, LeBlanc SA, Tonegawa S (2017) Basolateral to central amygdala neural circuits for appetitive behaviors. Neuron 93:1464-1479.e5.

King T, Vera-Portocarrero L, Gutierrez T, Vanderah TW, Dussor G, Lai J, Fields HL, Porreca F (2009) Unmasking the tonic-aversive state in neuropathic pain. Nat Neurosci 12:1364-1366.

Klapoetke NC, Murata Y, Kim SS, Pulver SR, Birdsey-Benson A, Cho YK, Morimoto TK, Chuong AS, Carpenter EJ, Tian Z, Wang J, Xie Y, Yan Z,
Zhang Y, Chow BY, Surek B, Melkonian M, Jayaraman V, ConstantinePaton M, Wong GK, et al. (2014) Independent optical excitation of distinct neural populations. Nat Methods 11:338-346.

Liu Q, Tang Z, Surdenikova L, Kim S, Patel KN, Kim A, Ru F, Guan Y, Weng HJ, Geng Y, Undem BJ, Kollarik M, Chen ZF, Anderson DJ, Dong X (2009) Sensory neuron-specific GPCR mrgprs are itch receptors mediating chloroquine-induced pruritus. Cell 139:1353-1365.

Lu YC, Chen YZ, Wei YY, He XT, Li X, Hu W, Yanagawa Y, Wang W, Wu SX, Dong YL (2015) Neurochemical properties of the synapses between the parabrachial nucleus-derived CGRP-positive axonal terminals and the GABAergic neurons in the lateral capsular division of central nucleus of amygdala. Mol Neurobiol 51:105-118.

Mu D, Sun YG (2017) Itch induces conditioned place aversion in mice. Neurosci Lett 658:91-96.

Mu D, Deng J, Liu KF, Wu ZY, Shi YF, Guo WM, Mao QQ, Liu XJ, Li H, Sun YG (2017) A central neural circuit for itch sensation. Science 357: 695-699.

Neugebauer V (2015) Amygdala pain mechanisms. Handb Exp Pharmacol 227:261-284.

Papoiu AD, Coghill RC, Kraft RA, Wang H, Yosipovitch G (2012) A tale of two itches: common features and notable differences in brain activation evoked by cowhage and histamine induced itch. Neuroimage 59:3611-3623.

Papoiu AD, Nattkemper LA, Sanders KM, Kraft RA, Chan YH, Coghill RC, Yosipovitch G (2013) Brain's reward circuits mediate itch relief: a functional MRI study of active scratching. PLoS One 8:e82389.

Post AM, Weyers P, Holzer P, Painsipp E, Pauli P, Wultsch T, Reif A, Lesch KP (2011) Gene-environment interaction influences anxiety-like behavior in ethologically based mouse models. Behav Brain Res 218:99-105.

Rodgers RJ, Johnson NJ (1995) Factor analysis of spatiotemporal and ethological measures in the murine elevated plus-maze test of anxiety. Pharmacol Biochem Behav 52:297-303.

Rothman S (1941) Physiology of itching. Physiol Rev 21:357-381.

Sanders KM, Akiyama T (2018) The vicious cycle of itch and anxiety. Neurosci Biobehav Rev 87:17-26.

Schneider G, Driesch G, Heuft G, Evers S, Luger TA, Ständer S (2006) Psychosomatic cofactors and psychiatric comorbidity in patients with chronic itch. Clin Exp Dermatol 31:762-767.

Schut C, Weik U, Tews N, Gieler U, Deinzer R, Kupfer J (2015) Coping as mediator of the relationship between stress and itch in patients with atopic dermatitis: a regression and mediation analysis. Exp Dermatol 24: $148-150$.

Shackman AJ, Salomons TV, Slagter HA, Fox AS, Winter JJ, Davidson RJ (2011) The integration of negative affect, pain and cognitive control in the cingulate cortex. Nat Rev Neurosci 12:154-167.

Simone DA, Nolano M, Johnson T, Wendelschafer-Crabb G, Kennedy WR (1998) Intradermal injection of capsaicin in humans produces degeneration and subsequent reinnervation of epidermal nerve fibers: correlation with sensory function. J Neurosci 18:8947-8959.

Takahashi A, Kato K, Makino J, Shiroishi T, Koide T (2006) Multivariate analysis of temporal descriptions of open-field behavior in wild-derived mouse strains. Behav Genet 36:763-774.

Vierow V, Forster C, Vogelgsang R, Dörfler A, Handwerker HO (2015) Cerebral networks linked to itch-related sensations induced by histamine and capsaicin. Acta Derm Venereol 95:645-652.

Vogt BA, Paxinos G (2014) Cytoarchitecture of mouse and rat cingulate cortex with human homologies. Brain Struct Funct 219:185-192.

Xiu J, Zhang Q, Zhou T, Zhou TT, Chen Y, Hu H (2014) Visualizing an emotional valence map in the limbic forebrain by TAI-FISH. Nat Neurosci 17:1552-1559. 